\title{
Widespread formation of cherts during the early Eocene climate optimum
}

\author{
Giovanni Muttoni $^{\mathrm{a}, *}$, Dennis V. Kent ${ }^{\mathrm{b}}$ \\ a Department of Earth Sciences, University of Milan, via Mangiagalli 34, I-20133 Milan, Italy, and ALP-Alpine Laboratory of Paleomagnetism, \\ via Madonna dei Boschi 76, I-12016 Peveragno (CN), Italy \\ ${ }^{\mathrm{b}}$ Department of Geological Sciences, Rutgers University, Piscataway, New Jersey 08854, USA, and Lamont-Doherty Earth Observatory, \\ Palisades, New York 10964, USA
}

Received 7 February 2007; received in revised form 25 May 2007; accepted 6 June 2007

\begin{abstract}
Radiolarian cherts in the Tethyan realm of Jurassic age were recently interpreted as resulting from high biosiliceous productivity along upwelling zones in subequatorial paleolatitudes the locations of which were confirmed by revised paleomagnetic estimates. However, the widespread occurrence of cherts in the Eocene suggests that cherts may not always be reliable proxies of latitude and upwelling zones. In a new survey of the global spatio-temporal distribution of Cenozoic cherts in Deep Sea Drilling Project (DSDP) and Ocean Drilling Program (ODP) sediment cores, we found that cherts occur most frequently in the Paleocene and early Eocene, with a peak in occurrences at $\sim 50 \mathrm{Ma}$ that is coincident with the time of highest bottom water temperatures of the early Eocene climatic optimum (EECO) when the global ocean was presumably characterized by reduced upwelling efficiency and biosiliceous productivity. Cherts occur less commonly during the subsequent Eocene global cooling trend. Primary paleoclimatic factors rather than secondary diagenetic processes seem therefore to control chert formation. This timing of peak Eocene chert occurrence, which is supported by detailed stratigraphic correlations, contradicts currently accepted models that involve an initial loading of large amounts of dissolved silica from enhanced weathering and/or volcanism in a supposedly sluggish ocean of the EECO, followed during the subsequent middle Eocene global cooling by more vigorous oceanic circulation and consequent upwelling that made this silica reservoir available for enhanced biosilicification, with the formation of chert as a result of biosilica transformation during diagenesis. Instead, we suggest that basin-basin fractionation by deep-sea circulation could have raised the concentration of EECO dissolved silica especially in the North Atlantic, where an alternative mode of silica burial involving widespread direct precipitation and/or absorption of silica by clay minerals could have been operative in order to maintain balance between silica input and output during the upwelling-deficient conditions of the EECO. Cherts may therefore not always be proxies of biosiliceous productivity associated with latitudinally focused upwelling zones.
\end{abstract}

(C) 2007 Elsevier B.V. All rights reserved.

Keywords: Chert; DSDP; ODP; Biosiliceous; Palygorskite; Cenozoic; Paleolatitude; Climate; Early Eocene climatic optimum

\footnotetext{
* Corresponding author.

E-mail address: giovanni.muttoni1@unimi.it (G. Muttoni).
}

\section{Introduction}

A paleomagnetic test on a presumed latitude-proxy facies was recently performed by analyzing radiolarian cherts in Jurassic sediments of the Tethyan realm (Muttoni et al., 2005). The premise was that the geographic 
distribution of planktonic organisms in the Jurassic followed actualistic patterns whereby biosiliceous organisms tend to proliferate in modern oceans in equatorial upwelling regions (e.g., Seibold and Berger, 1993). Deposition at near-equatorial paleolatitudes was confirmed by paleomagnetic data for the Tethyan Jurassic cherts (Muttoni et al., 2005). Cherts derived from the diagenetic transformation of silica-secreting organisms were therefore considered to mark the location of biosiliceous sediments in past high fertility (zonal) upwelling regions and tracked the motion of underlying plates (Berger and Winterer, 1974). This would be in line with previous observations of late Paleozoic, Jurassic, and Cretaceous cherts occurring at low paleolatitudes in the Pacific Ocean region (Larson et al., 1992; Hagstrum and Murchey, 1993; Richards et al., 1993).

These conclusions are challenged by the widespread geographic occurrence of Eocene chert. Chert, opaline claystone, porcellanite and related rock types of a common restricted age have long been recognized to occur throughout the western North Atlantic region and its margin and were encountered since the early days of ocean drilling within sediments of varied composition, environments of deposition, geologic settings, and burial depths (e.g., Gartner, 1970; Gibson and Towe, 1971; Riech and von Rad, 1979). These chert layers apparently correspond to acoustic horizon $\mathrm{A}^{\mathrm{c}}$, which can be traced as a seismic reflector across virtually the entire latitudinal extent of the western and eastern basins of the North Atlantic although in some areas it can also correspond to an erosional unconformity (Gartner, 1970; Tucholke and Mountain, 1979; Tucholke, 1979; Norris et al., 2001). This horizon was correlated to discrete stratigraphic levels straddling in toto biozones NP13NP15b and P9-P10 (Premoli Silva and Boersma, 1986; Aubry, 1995), i.e., latest early Eocene to early middle Eocene, or 45-50 Ma using the time scale of Berggren et al. (1995). The geographically widespread yet closely age correlative nature of the siliceous deposits from varied depositional environments could be explained by volcanic origin (Gibson and Towe, 1971; Mattson and Pessagno, 1971), although traces of siliceous microfossils suggested that most of these deposits actually represent highly altered diatomites (Wise and Weaver, 1973; Weaver and Wise, 1974). A complicated paleocirculation model was proposed by Ramsay (1971) to explain the apparently widespread high biosiliceous productivity needed to account for the vast area of Eocene cherts. The "silica burp" hypothesis (McGowran, 1989) suggested that the deposition of silica, which accumulated in the warm, supposedly sluggish oceans from volcanism and enhanced weath- ering of the continents during the early Eocene, was triggered by a sharp cooling that stimulated oceanic upwelling and biosiliceous productivity in the middle Eocene. Such temporally focused but broadly distributed occurrence of chert would limit the paleolatitudinal significance of this facies and suggest alternate modes of chert formation.

Here we explore this apparent incongruence by documenting the spatio-temporal distribution of Cenozoic cherts and biosiliceous sediments in the world ocean and their relationship to global climate as approximated by the benthic oxygen isotope $\left(\delta^{18} \mathrm{O}\right)$ record (Zachos et al., 2001; Miller et al., 2005). A general expectation for a biosiliceous origin for cherts is that they should occur more commonly during colder intervals when ocean circulation and upwelling is more vigorous than in warm intervals (e.g., Kidder and Erwin, 2001). The Cenozoic includes a well-documented wide climate range to gauge this proposition, from minimal ice sheets and even polar warmth in the Paleocene and especially the early Eocene and the transition through the middle and late Eocene towards the development of large polar ice sheets from the early Oligocene to Pleistocene. Eocene cherts are the most recent widespread occurrence of this facies and it should thus be possible to place them in optimal chronostratigraphic, paleogeographic and paleoclimatic contexts for understanding the origins and spatio-temporal distribution of more ancient cherts.

\section{Materials and methods}

\subsection{Occurrence of chert}

In a recent compilation of biogenic silica abundance in the geologic record, Kidder and Erwin (2001) surveyed selected paleontological journals and tabulated occurrences of silica-replaced fossils throughout the Phaneozoic; data for bedded chert occurrences were taken from an important compilation by Hein and Parrish (1987). Temporal resolution was at the epoch level, for example, the Paleocene, Eocene and Oligocene epochs of the Paleogene period, which had the highest total number of occurrences of bedded cherts (although negligible occurrences of silica-replaced fossils) of any geologic period in the Phanerozoic. In concert with these prior compilations, we assume that the occurrences of chert and porcellanite are early diagenetic products and thus reflect marine silica sources at about the time of deposition.

To refine the temporal resolution and significantly expand the geographic coverage of data for the 
Cenozoic, we searched for occurrences of chert or porcellanite in DSDP and ODP cores from the global ocean (Legs 1-208). We focus on the Cenozoic record because data coverage in Mesozoic DSDP and ODP cores is unlikely to be diagnostic due to the small overall number of sites. Specifically, we searched for stratigraphic intervals bearing chert or porcellanite layers and/or nodules in DSDP or ODP core barrel lithologic descriptions, avoiding dubious core catcher data. We retained only those intervals provided with an accurate biostratigraphic age determination expressed mainly in terms of calcareous nannoplankton, sometimes foraminifera, or in a few instances radiolarian zonations. To avoid data redundancy, stratigraphic intervals were integrated at the site level; for example, two correlative intervals with chert from two holes at the same site define a single chert occurrence interval.

We catalogued a total of 162 chert intervals. Each interval was assigned a numerical age range using biostratigraphic zonations from the Berggren et al. (1995) timescale (Table 1; Fig. 1A). Intervals overlapping in time were summed; for example, DSDP Site 111 with chert occurrence ranging over $0.45-1.95 \mathrm{Ma}$ and DSDP Site 635 with chert over 1.77-3.09 Ma were tabulated as one interval with chert from 0.45 to $1.77 \mathrm{Ma}$, two intervals with chert from 1.77 to $1.95 \mathrm{Ma}$, and one interval with chert from 1.95 to $3.09 \mathrm{Ma}$. Extending this summing procedure to all 162 intervals produced a total of 67 time intervals distributed from 0 to $65 \mathrm{Ma}$ where chert occurred from a minimum of 0 to a maximum of 46 times (Fig. 1A). This is basically the same counting procedure employed by Kidder and Erwin (2001) for the bedded chert data tabulated by Hein and Parrish (1987), although in our case it was done at the biozone rather than series/epoch level. To smooth out potentially spurious effects generated by the summing procedure, the chert occurrences were integrated into a cumulative distribution curve that was resampled and finally differentiated at $2 \mathrm{Myr}$ intervals (Fig. 1B). The resulting smoothed histogram of chert occurrences bears a reassuring close resemblance to the raw histogram of chert occurrences (Fig. 1A).

\subsection{Occurrence of radiolarians, diatoms and nannoplankton}

The occurrences in Cenozoic sediments of radiolarians and diatoms, biosiliceous fossils that may represent precursors to cherts, as well as of calcareous nannoplankton for control, were estimated by counting the number of DSDP and ODP holes with stratigraphic sections that contain diatoms or radiolarians or nannoplankton in $2 \mathrm{~m}$.y. bins from 0 to $64 \mathrm{Ma}$, where each count is a DSDP or ODP hole that contains at least one sample with diatoms or radiolarians or nannoplankton in a specific time interval [the results of a search of the Neptune database (online) 2006 (Spencer-Cervato, 1999) were provided by C. Spencer-Cervato and J. Reed (2006, personal communication)]. There are a total of 810 counts of radiolarians, 456 counts of diatoms and 1294 counts of nannoplankton for the Cenozoic; all sets of counts have a similar distribution with the highest number of occurrences in one of the recent bins and generally decreasing numbers of counts with increasing age. The overall decrease in microfossil occurrences with increasing age in part reflects the chronostratigraphic coverage of DSDP and ODP holes, which over the 0-64 Ma interval decreases more or less exponentially by an order of magnitude as mostly a consequence of variable drilling depths in a layer-cake stratigraphy (Spencer-Cervato, 1998, 1999) (Fig. 1C). The occurrences of nannoplankton, radiolarians and diatoms parallel the relative decrease in chronostratigraphic coverage of DSDP and ODP holes back until around $48 \mathrm{Ma}$, prior to which the biosiliceous occurrence curves are virtually at null values whereas the nannonplankton occurrences continue to track with the DSDP and ODP hole stratigraphic distribution (Fig. 1C). This suggests that the marked reduction in biosiliceous occurrences prior to about $48 \mathrm{Ma}$ is unlikely to be simply an artifact of the DSDP and ODP hole stratigraphic distribution, although other factors that might influence the occurrences, such as hiatuses and variable preservation as a function of differences in sedimentation rate (Nigrini et al., 2006), cannot be excluded. The overall shape of the chert occurrence distribution (Fig. 1A, B), with highest occurrences at the older end, is even more unlikely to be an artifact of the holes distribution.

A simple correction for the relative distribution of chronostratigraphic coverage of DSDP and ODP holes confirms that the peak in chert occurrences at around $50 \mathrm{Ma}$ (Fig. 1D) and the apparently corresponding reduced numbers of biosiliceous occurrences (Fig. 1C) cannot be ascribed to data distribution artifacts. Moreover, the occurrences of nannoplankton, which have a very widespread distribution that is not restricted to upwelling regions, were not reduced during the same time interval (Fig. 1C). Although the peak in chert occurrences may be partly accounted for by low rates of deposition and/or the development of unconformities (e.g., Nigrini et al., 2006), and bearing in mind all the uncertainties associated with the databases used in this study (e.g., Spencer-Cervato, 1999), we suspect the existence of an important yet paradoxical feature of silica distribution in Cenozoic oceanic sediments, 
Table 1

Chert intervals in DSDP and ODP sites

\begin{tabular}{|c|c|c|c|c|c|c|c|c|c|c|c|}
\hline Site & $\begin{array}{l}\text { Long } \\
\left({ }^{\circ}\right)\end{array}$ & $\begin{array}{l}\text { Lat } \\
\left({ }^{\circ}\right)\end{array}$ & Location & Biozones & Key & $\begin{array}{l}\text { From } \\
(\mathrm{Ma})\end{array}$ & $\begin{array}{l}\text { To } \\
\text { (Ma) }\end{array}$ & $\begin{array}{l}\text { Mage } \\
(\mathrm{Ma})\end{array}$ & $\begin{array}{l}\text { Durantion } \\
\text { (Myr) }\end{array}$ & $\begin{array}{l}\text { Plong } \\
\left({ }^{\circ}\right)\end{array}$ & $\begin{array}{l}\text { Plat } \\
\left({ }^{\circ}\right)\end{array}$ \\
\hline 1094 & 5.13 & -53.18 & Antarctic Meteor Rise & NN19 & $*$ & 0.40 & 1.95 & 1.20 & 1.50 & 4.96 & -53.35 \\
\hline 111 & -46.37 & 50.43 & North Atlantic & NN19 & $*_{£ \S}$ & 0.40 & 1.95 & 1.20 & 1.50 & -46.28 & 50.20 \\
\hline 635 & -77.33 & 25.42 & North Atlantic & PL5-PL6 & $*$ & 1.77 & 3.09 & 2.43 & 1.32 & -77.16 & 25.09 \\
\hline 157 & -85.90 & -1.76 & South Pacific & NN11 & $* \S$ & 5.60 & 8.60 & 7.10 & 3.00 & -92.70 & -3.70 \\
\hline 473 & -107.06 & 20.97 & G. of California & NN11a-NN11b & $*$ & 6.60 & 8.60 & 7.60 & 2.00 & -103.51 & 18.37 \\
\hline 438 & 143.23 & 40.63 & North Pacific & NN9-NN11 & $* \S$ & 5.60 & 10.70 & 8.15 & 5.10 & 141.73 & 42.19 \\
\hline 1126 & 128.07 & -33.52 & South Pacific Australia & NN10 & $*^{3}$ & 8.60 & 9.40 & 9.00 & 0.80 & 124.05 & -36.68 \\
\hline 1134 & 127.27 & -33.53 & South Pacific Australia & $\sim=\mathrm{NN} 6$ & $*$ & 11.80 & 13.60 & 12.70 & 1.80 & 121.75 & -38.32 \\
\hline 33 & -127.50 & 39.47 & North Pacific & NN6 & $* \S$ & 11.80 & 13.60 & 12.70 & 1.80 & -21.58 & 34.45 \\
\hline 1256 & -91.93 & 6.73 & Guatemala basin & NN5-NN7 & $*^{3}$ & 10.90 & 15.60 & 13.25 & 4.70 & -90.66 & 1.28 \\
\hline 682 & -79.06 & -11.27 & South Pacific & $\sim=\mathrm{M} 5 \mathrm{a}-\mathrm{M} 7$ & $*$ & 12.70 & 16.40 & 14.55 & 3.70 & -78.54 & -14.22 \\
\hline 1132 & 127.60 & -33.32 & South Pacific Australia & NN4-NN6 & $*$ & 11.80 & 18.30 & 15.05 & 6.50 & 121.23 & -39.18 \\
\hline 1133 & 128.90 & -33.53 & South Pacific Australia & NN4-NN6 & $*$ & 11.80 & 18.30 & 15.05 & 6.50 & 122.63 & -39.40 \\
\hline 1131 & 128.48 & -33.33 & South Pacific Australia & NN4-NN6 & $*$ & 11.80 & 18.30 & 15.05 & 6.50 & 122.18 & -39.20 \\
\hline 1129 & 128.48 & -33.28 & South Pacific Australia & NN4-NN5 & $*$ & 13.60 & 18.30 & 15.95 & 4.70 & 121.80 & -39.62 \\
\hline 153 & -72.43 & 13.97 & Caribbean & NN4-NN5 & $* \S$ & 13.60 & 18.30 & 15.95 & 4.70 & -74.05 & 11.20 \\
\hline 116 & -15.92 & 57.50 & North Atlantic & NN4 & $* \S$ & 15.60 & 18.30 & 16.95 & 2.70 & -23.05 & 54.73 \\
\hline 173 & -125.45 & 39.96 & North Pacific & N6-N7 (=M3-M4) & $*$ & 16.40 & 18.80 & 17.60 & 2.40 & -117.53 & 33.77 \\
\hline 416 & -10.80 & 32.84 & North Atlantic & N6-N7 (=M3-M4) & $*$ & 16.40 & 18.80 & 17.60 & 2.40 & -15.74 & 30.02 \\
\hline 397 & -15.18 & 26.84 & North Atlantic & NN1-NN4 & $* \S$ & 15.60 & 23.90 & 19.75 & 8.30 & -20.56 & 23.64 \\
\hline 626 & -79.55 & 25.60 & North Atlantic & $\mathrm{NN} 2$ & $*$ & 19.00 & 23.20 & 21.10 & 4.20 & -78.08 & 22.93 \\
\hline 765 & 117.57 & -15.98 & South Indian & $\mathrm{CN} 1 \mathrm{c}$ & $*$ & 19.20 & 23.20 & 21.20 & 4.00 & 108.47 & -24.69 \\
\hline 758 & 90.36 & 5.38 & North Indian & CP19-CN1 & $*$ & 19.20 & 29.90 & 24.55 & 10.70 & 80.49 & -3.68 \\
\hline 627 & -78.29 & 27.64 & North Atlantic & NP25 & $*$ & 23.90 & 27.50 & 25.70 & 3.60 & -76.40 & 24.73 \\
\hline 516 & -35.28 & -30.28 & South Atlantic & $\mathrm{P} 21-\mathrm{NN} 1$ & $*$ & 23.20 & 29.40 & 26.30 & 6.20 & -32.20 & -33.46 \\
\hline 960 & -2.73 & 3.58 & North Atlantic & $\mathrm{CP} 19 \mathrm{a}-\mathrm{CP} 19 \mathrm{~b}$ & $*$ & 23.90 & 29.90 & 26.90 & 6.00 & -10.38 & -0.72 \\
\hline 318 & -146.86 & -14.83 & South Pacific & NP24-NP25 & $*$ & 23.90 & 29.90 & 26.90 & 6.00 & -132.44 & -24.04 \\
\hline 689 & 3.10 & -64.52 & South Atlantic & CP17-CP19 & $*$ & 23.90 & 32.30 & 28.10 & 8.40 & 2.83 & -65.16 \\
\hline 1130 & 127.60 & -33.42 & South Pacific Australia & NP23-NP25 & $*$ & 23.90 & 32.30 & 28.10 & 8.40 & 117.00 & -46.74 \\
\hline 1134 & 127.27 & -33.53 & South Pacific Australia & NP23-NP25 & $*$ & 23.90 & 32.30 & 28.10 & 8.40 & 116.60 & -46.86 \\
\hline 96 & -85.76 & 23.74 & Gulf of Mexico & $\mathrm{P} 21$ & $*$ & 27.10 & 29.40 & 28.25 & 2.30 & -83.91 & 21.32 \\
\hline 70 & -140.36 & 6.34 & North Pacific & $\mathrm{P} 21$ & $* \S$ & 27.10 & 29.40 & 28.25 & 2.30 & -126.09 & -3.04 \\
\hline 268 & 105.16 & -63.95 & Antarctic & NP22-NP25 & $* \S$ & 23.90 & 32.80 & 28.35 & 8.90 & 98.25 & -60.65 \\
\hline 71 & -140.32 & 4.47 & North Pacific & NP23-NP24 & $* \S$ & 27.50 & 32.30 & 29.90 & 4.80 & -125.34 & -5.42 \\
\hline 462 & 165.03 & 7.24 & North Pacific & NP23-P20 & $*$ & 29.40 & 32.30 & 30.85 & 2.90 & -179.32 & -1.03 \\
\hline 117 & -15.40 & 57.34 & North Atlantic & NP23 & $* \S$ & 29.90 & 32.30 & 31.10 & 2.40 & -25.40 & 53.29 \\
\hline 706 & 61.37 & -13.11 & South Indian & NP23 & $*$ & 29.90 & 32.30 & 31.10 & 2.40 & 53.44 & -16.43 \\
\hline 959 & -2.73 & 3.63 & North Atlantic & CP17-CP18 & $*$ & 29.90 & 32.30 & 31.10 & 2.40 & -11.65 & -1.31 \\
\hline 116 & -15.92 & 57.50 & North Atlantic & P19 & $* \S$ & 30.30 & 32.00 & 31.15 & 1.70 & -25.88 & 53.43 \\
\hline 1126 & 128.07 & -33.52 & South Pacific Australia & NP16-NN2 & $*$ & 19.00 & 43.40 & 31.20 & 24.40 & 116.88 & -48.61 \\
\hline 628 & -78.32 & 27.53 & North Atlantic & NP22 & $*$ & 32.30 & 32.80 & 32.55 & 0.50 & -76.02 & 24.48 \\
\hline 317 & -162.26 & -11.00 & South Pacific & NP22 & * & 32.30 & 32.80 & 32.55 & 0.50 & -146.76 & -21.89 \\
\hline 316 & -157.13 & 0.09 & North Pacific & NP21 & $*$ & 32.80 & 34.20 & 33.50 & 1.40 & -141.00 & -11.13 \\
\hline 1134 & 127.27 & -33.53 & South Pacific Australia & NP18-NP21 & $*$ & 32.80 & 37.00 & 34.90 & 4.20 & 114.82 & -51.28 \\
\hline 1128 & 127.58 & -34.38 & South Pacific Australia & NP18-NP21 & * & 32.80 & 37.00 & 34.90 & 4.20 & 115.31 & -52.12 \\
\hline 318 & -146.86 & -14.83 & South Pacific & NP19-20 & $*$ & 34.20 & 36.00 & 35.10 & 1.80 & -129.45 & -26.27 \\
\hline 416 & -10.80 & 32.84 & North Atlantic & NP20? & $*$ & 34.20 & 36.00 & 35.10 & 1.80 & -19.45 & 27.62 \\
\hline 762 & 112.25 & -19.89 & South Indian & NP18-NP20 & $*$ & 34.20 & 37.00 & 35.60 & 2.80 & 95.88 & -37.75 \\
\hline 1090 & 8.90 & -42.92 & Antarctic Meteor Rise & NP18-NP19-20 & $*$ & 34.20 & 37.00 & 35.60 & 2.80 & -2.88 & -48.88 \\
\hline 1218 & -135.36 & 8.88 & Central Pacific & CP15 & $*$ & 34.20 & 37.10 & 35.65 & 2.90 & -118.90 & -2.07 \\
\hline 703 & 7.89 & -47.05 & South Atlantic & NP18 & $*$ & 36.00 & 37.00 & 36.50 & 1.00 & -4.78 & -53.14 \\
\hline 317 & -162.26 & -11.00 & South Pacific & NP17-NP19-20 & $*$ & 34.20 & 40.40 & 37.30 & 6.20 & -145.32 & -23.29 \\
\hline 209 & 152.19 & -15.94 & Coral Sea & NP17 & $*$ & 37.00 & 40.40 & 38.70 & 3.40 & 141.09 & -31.76 \\
\hline 547 & -9.35 & 33.78 & North Atlantic & NP16-NP19-20 & $*$ & 34.20 & 43.40 & 38.80 & 9.20 & -19.00 & 28.01 \\
\hline 167 & -176.82 & 7.07 & North Pacific & $\mathrm{P} 14$ & $*$ & 38.40 & 40.10 & 39.25 & 1.70 & -159.05 & -5.12 \\
\hline 70 & -140.36 & 6.34 & North Pacific & P14 & $* \S$ & 38.40 & 40.10 & 39.25 & 1.70 & -122.67 & -5.81 \\
\hline 73 & -137.47 & -1.91 & South Pacific & P13-P14 & $*^{3}$ & 38.40 & 40.50 & 39.45 & 2.10 & -118.96 & -13.92 \\
\hline
\end{tabular}


Table 1 (continued)

\begin{tabular}{|c|c|c|c|c|c|c|c|c|c|c|c|}
\hline Site & $\begin{array}{l}\text { Long } \\
\left({ }^{\circ}\right)\end{array}$ & $\begin{array}{l}\text { Lat } \\
\left(^{\circ}\right)\end{array}$ & Location & Biozones & Key & $\begin{array}{l}\text { From } \\
\text { (Ma) }\end{array}$ & $\begin{array}{l}\text { To } \\
\text { (Ma) }\end{array}$ & $\begin{array}{l}\text { Mage } \\
\text { (Ma) }\end{array}$ & $\begin{array}{l}\text { Durantion } \\
\text { (Myr) }\end{array}$ & $\begin{array}{l}\text { Plong } \\
\left({ }^{\circ}\right)\end{array}$ & $\begin{array}{l}\text { Plat } \\
\left({ }^{\circ}\right)\end{array}$ \\
\hline 1052 & -76.63 & 29.95 & North Atlantic Blake Nose & P12-P13 & $*$ & 40.10 & 43.60 & 41.85 & 3.50 & -73.42 & 26.94 \\
\hline 702 & -26.37 & -50.95 & South Atlantic & NP16 & $*$ & 40.40 & 43.40 & 41.90 & 3.00 & -19.14 & -54.22 \\
\hline 707 & 59.02 & -7.55 & South Indian & NP15-NP17 & $*$ & 37.00 & 47.30 & 42.15 & 10.30 & 46.79 & -13.86 \\
\hline 313 & -170.95 & 20.17 & North Pacific & NP11-NP22 & $* \S$ & 32.30 & 53.61 & 42.96 & 21.31 & -151.79 & 6.22 \\
\hline 370 & -10.78 & 32.84 & North Atlantic & NP14a-NP17 & $*_{£}$ & 37.00 & 49.70 & 43.35 & 12.70 & -21.35 & 27.14 \\
\hline 366 & -19.85 & 5.68 & North Atlantic & NP14-NP17 & $*_{£}$ & 37.00 & 49.70 & 43.35 & 12.70 & -32.93 & 0.95 \\
\hline 292 & 124.65 & 15.82 & Philippine Sea & NP12-NP20 & $* \S$ & 34.20 & 52.85 & 43.53 & 18.65 & 146.46 & 10.88 \\
\hline 738 & 82.79 & -62.71 & South Antarctic & CP13-CP14a & $*$ & 40.40 & 47.30 & 43.85 & 6.90 & 72.64 & -61.02 \\
\hline 318 & -146.86 & -14.83 & South Pacific & NP15-NP16 & $*$ & 40.40 & 47.30 & 43.85 & 6.90 & -126.47 & -28.71 \\
\hline 703 & 7.89 & -47.05 & South Atlantic & NP15-NP16 & $*$ & 40.40 & 47.30 & 43.85 & 6.90 & -10.00 & -53.87 \\
\hline 1183 & 157.02 & -1.18 & Pacific Ontong Java & NP9-NP22 & $*$ & 32.30 & 56.20 & 44.25 & 23.90 & 174.39 & -12.64 \\
\hline 530 & 9.39 & -19.19 & South Atlantic & NP15 & $* \S £$ & 43.40 & 47.30 & 45.35 & 3.90 & -6.25 & -26.24 \\
\hline 749 & 76.41 & -58.72 & South Indian & NP13-NP16 & $*$ & 40.40 & 50.60 & 45.50 & 10.20 & 65.33 & -57.25 \\
\hline 748 & 79.00 & -58.44 & South Indian & NP13-NP16 & $*$ & 40.40 & 50.60 & 45.50 & 10.20 & 67.80 & -56.93 \\
\hline 387 & -67.67 & 32.32 & North Atlantic & $\sim \mathrm{NP} 15 \mathrm{a}-\mathrm{NP} 15 \mathrm{~b}$ & $*_{£}$ & 44.50 & 47.30 & 45.90 & 2.80 & -64.25 & 28.85 \\
\hline 219 & 72.88 & 9.03 & Arabian Sea & P10-P11 & $*$ & 43.60 & 49.00 & 46.30 & 5.40 & 53.25 & -7.31 \\
\hline 761 & 115.54 & -16.74 & South Indian & NP14-NP15 & $*$ & 43.40 & 49.70 & 46.55 & 6.30 & 95.68 & -37.17 \\
\hline 70 & -140.36 & 6.34 & North Pacific & NP14-NP15 & $* \S$ & 43.40 & 49.70 & 46.55 & 6.30 & -122.31 & -7.84 \\
\hline 13 & -18.23 & 6.04 & North Atlantic & NP14-NP15 & $* \S$ & 43.40 & 49.70 & 46.55 & 6.30 & -32.41 & 1.40 \\
\hline 264 & 112.04 & -34.97 & Indian & NP14-NP15 & $*$ & 43.40 & 49.70 & 46.55 & 6.30 & 88.54 & -55.01 \\
\hline 208 & 161.22 & -26.11 & Tasman Sea & NP14-NP15 & $* \S$ & 43.40 & 49.70 & 46.55 & 6.30 & 152.52 & -41.75 \\
\hline 207 & 165.43 & -36.96 & Tasman Sea & NP12-NP16 & $* \S$ & 40.40 & 52.85 & 46.62 & 12.45 & 162.50 & -50.89 \\
\hline 959 & -2.73 & 3.63 & North Atlantic & $\mathrm{CP} 8 \mathrm{a}-\mathrm{CP} 14 \mathrm{~b}$ & $*$ & 37.10 & 56.20 & 46.65 & 19.10 & -17.34 & -2.58 \\
\hline 1222 & -143.88 & 13.82 & Central Pacific & $\mathrm{CP} 9 \mathrm{a}-\mathrm{RP} 15 \sim=\mathrm{CP} 9 \mathrm{a}-\mathrm{P} 14$ & $*$ & 38.40 & 55.00 & 46.70 & 16.60 & -126.76 & -0.94 \\
\hline 1217 & -138.10 & 16.87 & Central Pacific & NP10-RP14 =NP10-CP14a & $*$ & 40.40 & 55.00 & 47.70 & 14.60 & -122.03 & 2.32 \\
\hline 585 & 156.82 & 13.48 & North Pacific & $\mathrm{P} 7-\mathrm{P} 11$ & $*$ & 43.60 & 52.30 & 47.95 & 8.70 & 175.42 & -1.08 \\
\hline 462 & 165.03 & 7.24 & North Pacific & NP12-NP15 & $*$ & 43.40 & 52.85 & 48.12 & 9.45 & -177.53 & -8.63 \\
\hline 317 & -162.26 & -11.00 & South Pacific & NP12-NP15 & $*$ & 43.40 & 52.85 & 48.12 & 9.45 & -143.72 & -28.10 \\
\hline 1050 & -76.23 & 30.10 & North Atlantic Blake Nose & CP11a-CP13a & $*$ & 46.10 & 50.60 & 48.35 & 4.50 & -72.66 & 27.82 \\
\hline 552 & -23.23 & 56.04 & North Atlantic & NP14 & $* \S £$ & 47.30 & 49.70 & 48.50 & 2.40 & -35.23 & 51.55 \\
\hline 106 & -69.46 & 36.43 & North Atlantic & NP14 & $*$ & 47.30 & 49.70 & 48.50 & 2.40 & -65.18 & 33.39 \\
\hline 1216 & -139.48 & 21.45 & Central Pacific & RP9-RP11 = P9-CP12 & $*$ & 47.30 & 50.40 & 48.85 & 3.10 & -124.49 & 6.09 \\
\hline 386 & -64.25 & 31.19 & North Atlantic & NP13-NP14b & $*_{£}$ & 47.30 & 50.60 & 48.95 & 3.30 & -61.01 & 27.56 \\
\hline 1136 & 84.83 & -59.65 & Antarctic Kerguelen & $\mathrm{CP} 9 \mathrm{~b}-\mathrm{CP} 13 \mathrm{~b}$ & $*$ & 44.50 & 53.61 & 49.06 & 9.11 & 71.42 & -57.90 \\
\hline 765 & 117.57 & -15.98 & South Indian & $\mathrm{CP} 12 \mathrm{a}$ & $*$ & 48.50 & 49.70 & 49.10 & 1.20 & 96.88 & -36.76 \\
\hline 612 & -72.77 & 38.82 & North Atlantic & NP14a & $*_{£}$ & 48.50 & 49.70 & 49.10 & 1.20 & -67.91 & 36.17 \\
\hline 1049 & -76.12 & 30.15 & North Atlantic Blake Nose & $\mathrm{CP} 12 \mathrm{a}$ & $*$ & 48.50 & 49.70 & 49.10 & 1.20 & -72.51 & 27.95 \\
\hline 390 & -76.11 & 30.14 & North Atlantic & NP14a & $*_{£}$ & 48.50 & 49.70 & 49.10 & 1.20 & -72.50 & 27.94 \\
\hline 960 & -2.73 & 3.58 & North Atlantic & CP10-CP13a & $*$ & 46.10 & 52.85 & 49.47 & 6.75 & -18.27 & -2.55 \\
\hline 98 & -77.31 & 25.38 & North Atlantic & P9 & $*$ & 49.00 & 50.40 & 49.70 & 1.40 & -74.31 & 23.44 \\
\hline 206 & 165.45 & -32.01 & Tasman Sea & NP12-NP14 & $* \S$ & 47.30 & 52.85 & 50.07 & 5.55 & 158.61 & -47.06 \\
\hline 1220 & -142.77 & 10.18 & Central Pacific & $\mathrm{RP} 8-\mathrm{RP} 11 \sim=\mathrm{CP} 10-\mathrm{CP} 12$ & $*$ & 47.30 & 52.85 & 50.07 & 5.55 & -126.10 & -6.06 \\
\hline 356 & -41.09 & -28.29 & South Atlantic & NP13 & $*_{£}$ & 49.70 & 50.60 & 50.15 & 0.90 & -37.81 & -32.10 \\
\hline 384 & -51.66 & 40.36 & North Atlantic & NP13 & $* £$ & 49.70 & 50.60 & 50.15 & 0.90 & -47.63 & 35.71 \\
\hline 316 & -157.13 & 0.09 & North Pacific & NP11-NP14 & $*$ & 47.30 & 53.61 & 50.46 & 6.31 & -139.39 & -18.12 \\
\hline 219 & 72.88 & 9.03 & Arabian Sea & P8 & $*$ & 50.40 & 50.80 & 50.60 & 0.40 & 50.71 & -10.89 \\
\hline 613 & -72.52 & 38.77 & North Atlantic & NP12-NP14a & $* £$ & 48.50 & 52.85 & 50.67 & 4.35 & -67.45 & 36.19 \\
\hline 1186 & 159.85 & -0.68 & Pacific Ontong Java & NP1-NP17 & $*$ & 37.00 & 65.00 & 51.00 & 28.00 & 175.16 & -17.76 \\
\hline 404 & -23.25 & 56.05 & North Atlantic & NP12-NP13 & $*_{£}$ & 49.70 & 52.85 & 51.28 & 3.15 & -36.19 & 51.25 \\
\hline 628 & -78.32 & 27.53 & North Atlantic & P7-P8 & $*$ & 50.40 & 52.30 & 51.35 & 1.90 & -74.77 & 25.73 \\
\hline 1135 & 84.27 & -59.70 & Antarctic Kerguelen & CP4-CP12/13 & $*$ & 43.40 & 59.70 & 51.55 & 16.30 & 70.05 & -57.81 \\
\hline 1221 & -143.70 & 12.03 & Central Pacific & $\mathrm{P} 5-\mathrm{RP} 11 \sim=\mathrm{P} 5-\mathrm{CP} 12$ & $*$ & 47.30 & 55.90 & 51.60 & 8.60 & -127.72 & -4.94 \\
\hline 612 & -72.77 & 38.82 & North Atlantic & NP12 & $*_{£}$ & 50.60 & 52.85 & 51.72 & 2.25 & -67.44 & 36.23 \\
\hline 387 & -67.67 & 32.32 & North Atlantic & NP12 & $*_{£}$ & 50.60 & 52.85 & 51.72 & 2.25 & -63.67 & 29.20 \\
\hline 405 & -22.06 & 55.34 & North Atlantic & NP12 & $*_{£}$ & 50.60 & 52.85 & 51.72 & 2.25 & -35.10 & 50.53 \\
\hline 698 & -33.10 & -51.46 & South Atlantic & NP12 & $*$ & 50.60 & 52.85 & 51.72 & 2.25 & -26.93 & -54.98 \\
\hline 111 & -46.37 & 50.43 & North Atlantic & NP12 & $*_{£ \S}$ & 50.60 & 52.85 & 51.72 & 2.25 & -40.82 & 45.02 \\
\hline
\end{tabular}


Table 1 (continued)

\begin{tabular}{|c|c|c|c|c|c|c|c|c|c|c|c|}
\hline Site & $\begin{array}{l}\text { Long } \\
\left({ }^{\circ}\right)\end{array}$ & $\begin{array}{l}\text { Lat } \\
\left({ }^{\circ}\right)\end{array}$ & Location & Biozones & Key & $\begin{array}{l}\text { From } \\
(\mathrm{Ma})\end{array}$ & $\begin{array}{l}\text { To } \\
\text { (Ma) }\end{array}$ & $\begin{array}{l}\text { Mage } \\
\text { (Ma) }\end{array}$ & $\begin{array}{l}\text { Durantion } \\
\text { (Myr) }\end{array}$ & $\begin{array}{l}\text { Plong } \\
\left({ }^{\circ}\right)\end{array}$ & $\begin{array}{l}\text { Plat } \\
\left({ }^{\circ}\right)\end{array}$ \\
\hline ISL.B. & -74.08 & 39.80 & North Atlantic & NP12 & $* \circ$ & 50.60 & 52.85 & 51.72 & 2.25 & -68.51 & 37.35 \\
\hline 961 & -3.67 & 3.45 & North Atlantic & CP10 & $*$ & 50.60 & 52.85 & 51.72 & 2.25 & -19.90 & -2.93 \\
\hline 750 & 81.24 & -57.59 & South Indian & NP12 & $*$ & 50.60 & 52.85 & 51.72 & 2.25 & 67.10 & -55.73 \\
\hline 627 & -78.29 & 27.64 & North Atlantic & NP12 & $*$ & 50.60 & 52.85 & 51.72 & 2.25 & -74.67 & 25.84 \\
\hline 762 & 112.25 & -19.89 & South Indian & NP11-NP12 & $*$ & 50.60 & 53.61 & 52.10 & 3.01 & 88.75 & -40.31 \\
\hline 738 & 82.79 & -62.71 & South Antarctic & CP8b-CP11 & $*$ & 49.70 & 55.16 & 52.43 & 5.46 & 68.67 & -60.81 \\
\hline 702 & -26.37 & -50.95 & South Atlantic & NP9b-NP12 & $*$ & 50.60 & 55.16 & 52.88 & 4.56 & -19.95 & -54.27 \\
\hline 707 & 59.02 & -7.55 & South Indian & NP9-NP13 & $*$ & 49.70 & 56.20 & 52.95 & 6.50 & 43.01 & -16.04 \\
\hline 700 & -30.28 & -51.53 & South Atlantic & NP9-NP12 & $*$ & 50.60 & 56.20 & 53.40 & 5.60 & -24.15 & -55.21 \\
\hline 146 & -69.38 & 55.34 & Caribbean & NP9-NP12 & $* \S$ & 50.60 & 56.20 & 53.40 & 5.60 & -79.28 & 11.74 \\
\hline 119 & -7.97 & 45.03 & North Atlantic & NP9-NP12 & $* \S$ & 50.60 & 56.20 & 53.40 & 5.60 & -20.72 & 40.55 \\
\hline 98 & -77.31 & 25.38 & North Atlantic & P6 & $*$ & 52.30 & 54.70 & 53.50 & 2.40 & -73.79 & 23.47 \\
\hline 96 & -85.76 & 23.74 & Gulf of Mexico & P6 & $*$ & 52.30 & 54.70 & 53.50 & 2.40 & -82.33 & 23.00 \\
\hline 117 & -15.40 & 57.34 & North Atlantic & NP10 & $* \S$ & 53.61 & 55.00 & 54.31 & 1.39 & -29.15 & 51.85 \\
\hline 1050 & -76.23 & 30.10 & North Atlantic Blake Nose & $\mathrm{CP} 8 \mathrm{~b}-\mathrm{CP} 9 \mathrm{a}$ & $*$ & 53.61 & 55.16 & 54.39 & 1.55 & -71.80 & 27.99 \\
\hline 748 & 79.00 & -58.44 & South Indian & NP9-NP12 & $*$ & 52.85 & 56.20 & 54.53 & 3.35 & 64.50 & -56.70 \\
\hline 752 & 93.58 & -30.89 & South Indian & СР8-CP9 & $*$ & 52.85 & 56.20 & 54.53 & 3.35 & 61.77 & -47.94 \\
\hline 547 & -9.35 & 33.78 & North Atlantic & NP5-NP13 & $*$ & 49.70 & 59.70 & 54.70 & 10.00 & -22.57 & 27.10 \\
\hline 152 & -74.61 & 15.88 & Caribbean & NP9-NP10 & $* \S$ & 53.61 & 56.20 & 54.90 & 2.59 & -84.51 & 11.21 \\
\hline 634 & -77.32 & 25.38 & North Atlantic & $\mathrm{NP9b}$ & $*$ & 55.00 & 55.16 & 55.08 & 0.16 & -73.56 & 23.47 \\
\hline 1001 & -74.92 & 15.75 & North Atlantic Caribbean & CP4-CP10 & $*$ & 50.60 & 59.70 & 55.15 & 9.10 & -84.76 & 10.90 \\
\hline 1265 & 2.63 & -28.83 & South Atlantic Walvis Ridge & СР6-CP9a & $*$ & 53.61 & 57.50 & 55.56 & 3.89 & -18.77 & -36.58 \\
\hline 316 & -157.13 & 0.09 & North Pacific & NP9 & $*$ & 55.00 & 56.20 & 55.60 & 1.20 & -140.48 & -20.07 \\
\hline 698 & -33.10 & -51.46 & South Atlantic & NP9 & $*$ & 55.00 & 56.20 & 55.60 & 1.20 & -27.66 & -55.70 \\
\hline 961 & -3.67 & 3.45 & North Atlantic & CP6-CP8a & $*$ & 55.16 & 57.50 & 56.33 & 2.34 & -21.44 & -3.97 \\
\hline 153 & -72.43 & 13.97 & Caribbean & NP5-NP10 & $* \S$ & 53.61 & 59.70 & 56.65 & 6.09 & -81.71 & 9.84 \\
\hline 217 & 90.54 & 8.93 & Indian Ocean & NP5-NP10 & $* \S$ & 53.61 & 59.70 & 56.65 & 6.09 & 68.80 & -8.91 \\
\hline 390 & -76.11 & 30.14 & North Atlantic & NP7-NP8 & $*_{£}$ & 56.20 & 57.50 & 56.85 & 1.30 & -71.26 & 27.97 \\
\hline 1049 & -76.12 & 30.15 & North Atlantic Blake Nose & CP6 & $*$ & 56.90 & 57.50 & 57.20 & 0.60 & -71.18 & 27.98 \\
\hline 702 & -26.37 & -50.95 & South Atlantic & NP6-NP7 & $*$ & 57.30 & 58.40 & 57.85 & 1.10 & -20.74 & -55.27 \\
\hline 207 & 165.43 & -36.96 & Tasman Sea & NP6-NP7 & $* \S$ & 57.30 & 58.40 & 57.85 & 1.10 & 159.55 & -51.50 \\
\hline 465 & 178.92 & 33.82 & North Pacific & NP5-NP8 & $*$ & 56.20 & 59.70 & 57.95 & 3.50 & -165.67 & 11.49 \\
\hline 530 & 9.39 & -19.19 & South Atlantic & NP3-NP10 & $* \xi £$ & 53.61 & 63.80 & 58.71 & 10.19 & -11.36 & -28.42 \\
\hline 208 & 161.22 & -26.11 & Tasman Sea & NP3-NP10 & $* \S$ & 53.61 & 63.80 & 58.71 & 10.19 & 148.97 & -42.57 \\
\hline 702 & -26.37 & -50.95 & South Atlantic & $\mathrm{P} 3 \mathrm{~b}$ & $*$ & 59.20 & 60.00 & 59.60 & 0.80 & -21.04 & -55.62 \\
\hline 698 & -33.10 & -51.46 & South Atlantic & NP4-NP7 & $*$ & 57.30 & 62.20 & 59.75 & 4.90 & -28.50 & -56.45 \\
\hline 750 & 81.24 & -57.59 & South Indian & NP4-NP6 & $*$ & 57.50 & 62.20 & 59.85 & 4.70 & 65.65 & -56.06 \\
\hline 206 & 165.45 & -32.01 & Tasman Sea & NP3-NP8? & $* \S$ & 56.20 & 63.80 & 60.00 & 7.60 & 156.49 & -46.91 \\
\hline 758 & 90.36 & 5.38 & North Indian & $\mathrm{CP} 1 \mathrm{~b}-\mathrm{CP} 6$ & $*$ & 56.90 & 64.50 & 60.70 & 7.60 & 66.32 & -13.38 \\
\hline 1050 & -76.23 & 30.10 & North Atlantic Blake Nose & $\mathrm{CP} 3$ & $*$ & 59.70 & 62.20 & 60.95 & 2.50 & -70.65 & 27.91 \\
\hline 316 & -157.13 & 0.09 & North Pacific & NP3-NP5 & $*$ & 58.40 & 63.80 & 61.10 & 5.40 & -141.48 & -22.01 \\
\hline 1052 & -76.63 & 29.95 & North Atlantic Blake Nose & $\mathrm{P} 1 \mathrm{c}-\mathrm{P} 3 \mathrm{~b}$ & $*$ & 59.20 & 63.00 & 61.10 & 3.80 & -71.01 & 27.83 \\
\hline 738 & 82.79 & -62.71 & South Antarctic & $\mathrm{CP} 1-\mathrm{CP} 4$ & $*$ & 58.40 & 65.00 & 61.70 & 6.60 & 67.46 & -61.30 \\
\hline 752 & 93.58 & -30.89 & South Indian & $\mathrm{CP} 1-\mathrm{CP} 4$ & $*$ & 58.40 & 65.00 & 61.70 & 6.60 & 58.93 & -49.59 \\
\hline 700 & -30.28 & -51.53 & South Atlantic & NP3-NP4 & $*$ & 59.70 & 63.80 & 61.75 & 4.10 & -25.83 & -56.76 \\
\hline 462 & 165.03 & 7.24 & North Pacific & NP3-NP4 & $*$ & 59.70 & 63.80 & 61.75 & 4.10 & 178.21 & -15.91 \\
\hline 761 & 115.54 & -16.74 & South Indian & NP2-NP4 & $*$ & 59.70 & 64.50 & 62.10 & 4.80 & 90.17 & -39.26 \\
\hline 1049 & -76.12 & 30.15 & North Atlantic Blake Nose & $\mathrm{CP} 2$ & $*$ & 62.20 & 63.80 & 63.00 & 1.60 & -70.10 & 27.96 \\
\hline
\end{tabular}

Site $=$ DSDP or ODP sites with chert intervals (ISL. B. $=$ ODP Leg 150X Island Beach site). Long, Lat $=$ present-day longitude $\left({ }^{\circ} \mathrm{E}\right.$ when positive, ${ }^{\circ} \mathrm{W}$ when negative) and latitude $\left({ }^{\circ} \mathrm{N}\right.$ when positive, ${ }^{\circ} \mathrm{S}$ when negative) of DSDP or ODP sites with chert intervals. Location = geographic location of DSDP or ODP sites with chert intervals. Biozones = biostratigraphic zonation of chert intervals (NN, CN, NP, and CP are calcareous nannoplankton zonations; PL, M, and P are foraminifera zonations; RP is radiolaria zonation; for NN, CN, NP, CP, PL, M, and P see Berggren et al., 1995 and references therein, whereas for RP see Sanfilippo and Nigrini, 1998). Key = biostratigraphic attribution of chert intervals according to: * DSDP or ODP data from Proceedings or Preliminary Report volumes; £ Aubry (1995); § C. Agnini (pers. comm.); ${ }^{\circ}$ Van Fossen (Proceedings of the Ocean Drilling Program, Scientific Results 150X, pp. 295-304, 1997). From, To, Mage = younger limit age of chert intervals, older limit age of chert intervals, and mean age of chert intervals (in Ma) derived from the assigned biostratigraphic zonation referred to the Berggren et al. (1995) time scale. Dur. = duration of chert intervals expressed in Myr. Plong, Plat $=$ paleolongitude $\left({ }^{\circ} \mathrm{E}\right.$ when positive, ${ }^{\circ} \mathrm{W}$ when negative)) and paleolatitude $\left({ }^{\circ} \mathrm{N}\right.$ when positive, ${ }^{\circ} \mathrm{S}$ when negative) of DSDP or ODP sites with chert intervals (from Ocean Drilling Stratigraphic Network online; plate reconstruction data from Hay et al., 1999 and references therein). 
(A)

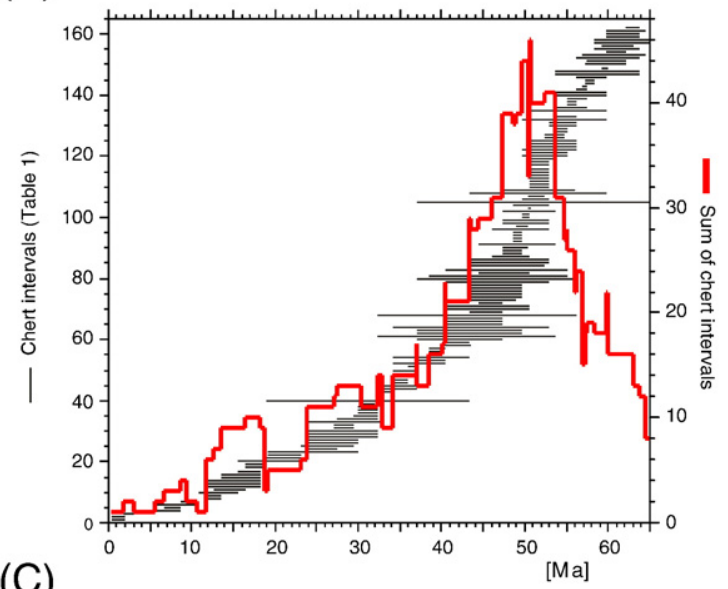

(C)

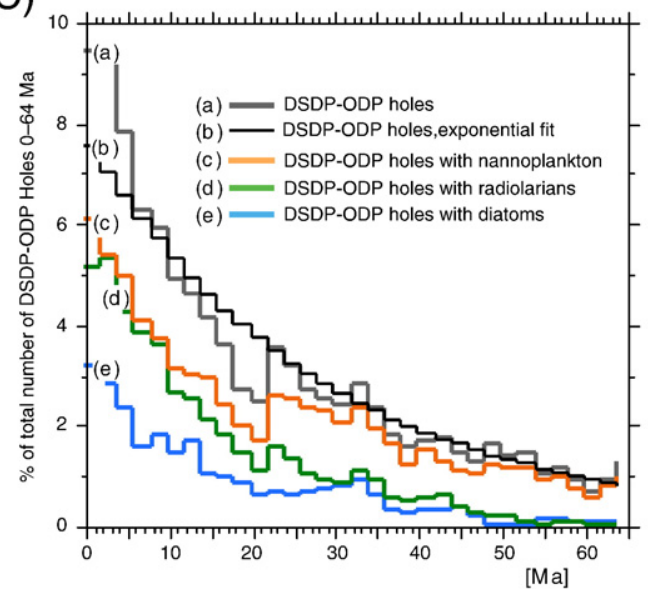

(B)

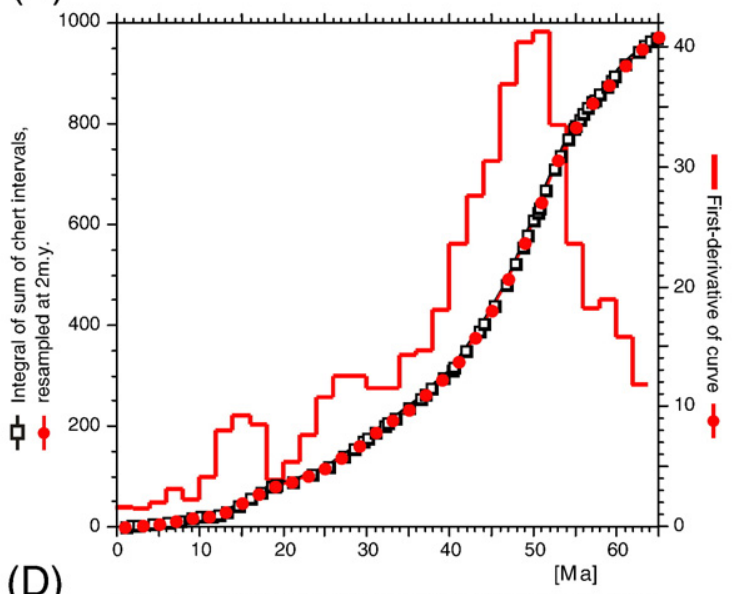

(D)

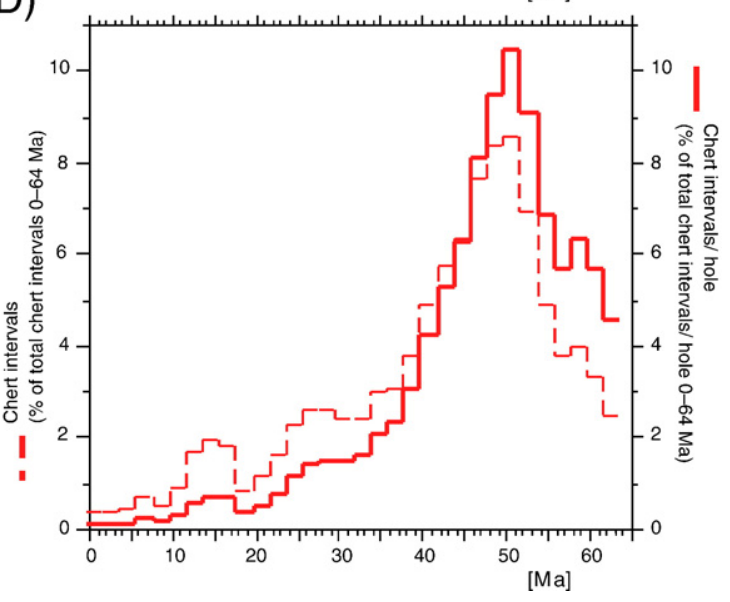

Fig. 1. (A) Temporal distribution of stratigraphic intervals with chert and summed number of biostratigraphic intervals with chert based on data from DSDP and ODP sites (Table 1). (B) Summed number of biostratigraphic intervals with chert integrated into a cumulative frequency curve that was resampled and differentiated at 2 Myr intervals. (C) Chronostratigraphic coverage of DSDP and ODP holes expressed as \% of the total number of DSDP and ODP holes present in the 0-64 Ma interval (curve labelled a, with exponential fit represented by curve b); chronostratigraphic coverage of DSDP and ODP holes with nannoplankton (curve c), radiolarians (curve d) and diatoms (curve e) expressed as \% of the total number of DSDP and ODP holes present in the 0-64 Ma interval; data from Neptune [online]; generated February 21, 2006 by Josh Reed using CHRONOS XML searches; http://services.chronos.org/databases/neptune/index.html. (D) The chronostratigraphic coverage of chert intervals (from panel B), here expressed as percentage of the total number of chert intervals recorded in the 0-64 Ma interval (histogram with dashed line), was corrected for an exponential fit to the chronostratigraphic coverage of DSDP and ODP holes (panel C, curve b); we proceeded as follows: the number of chert intervals in each 2 Myr bin was divided by the number of DSDP and ODP holes occurring in the same bin that resulted from the exponential fit applied to the original DSDP and ODP holes distribution (panel C, curve b). The resulting curve (histogram with thick line) represents the chronostratigraphic coverage of chert intervals per hole, which we express as \% of the total number of chert intervals per hole present in the 0-64 Ma interval. All ages estimated according to the Berggren et al. (1995) timescale.

whereby the highest occurrences of chert (i.e., from $\sim 46$ to $65 \mathrm{Ma}$ and peaking at $\sim 50 \mathrm{Ma}$ ) apparently correspond to the lowest occurrences of biosiliceous (radiolarian and diatom) fossils.

\section{Climatic and palaeogeographic setting of Eocene cherts}

The age frequency distribution of chert shows an unexpected positive correlation with ocean bottom water temperatures estimated from benthic foraminifera oxygen isotopes (Zachos et al., 2001; Miller et al., 2005) (Fig. 2). As indicated by oxygen isotopes, bottom waters warmed (i.e., $\delta^{18} \mathrm{O}$ decreased) over $\sim 10 \mathrm{Myr}$ of the late Paleocene and early Eocene to peak temperatures of around $14{ }^{\circ} \mathrm{C}$ in the early Eocene climatic optimum (EECO) centered at around $50 \mathrm{Ma}$. Chert occurrences also increased in the Paleocene and early Eocene and reached maximum occurrences in nannofossil Zones $\sim$ NP11-NP15a ( 54-46 Ma), bracketing the peak 


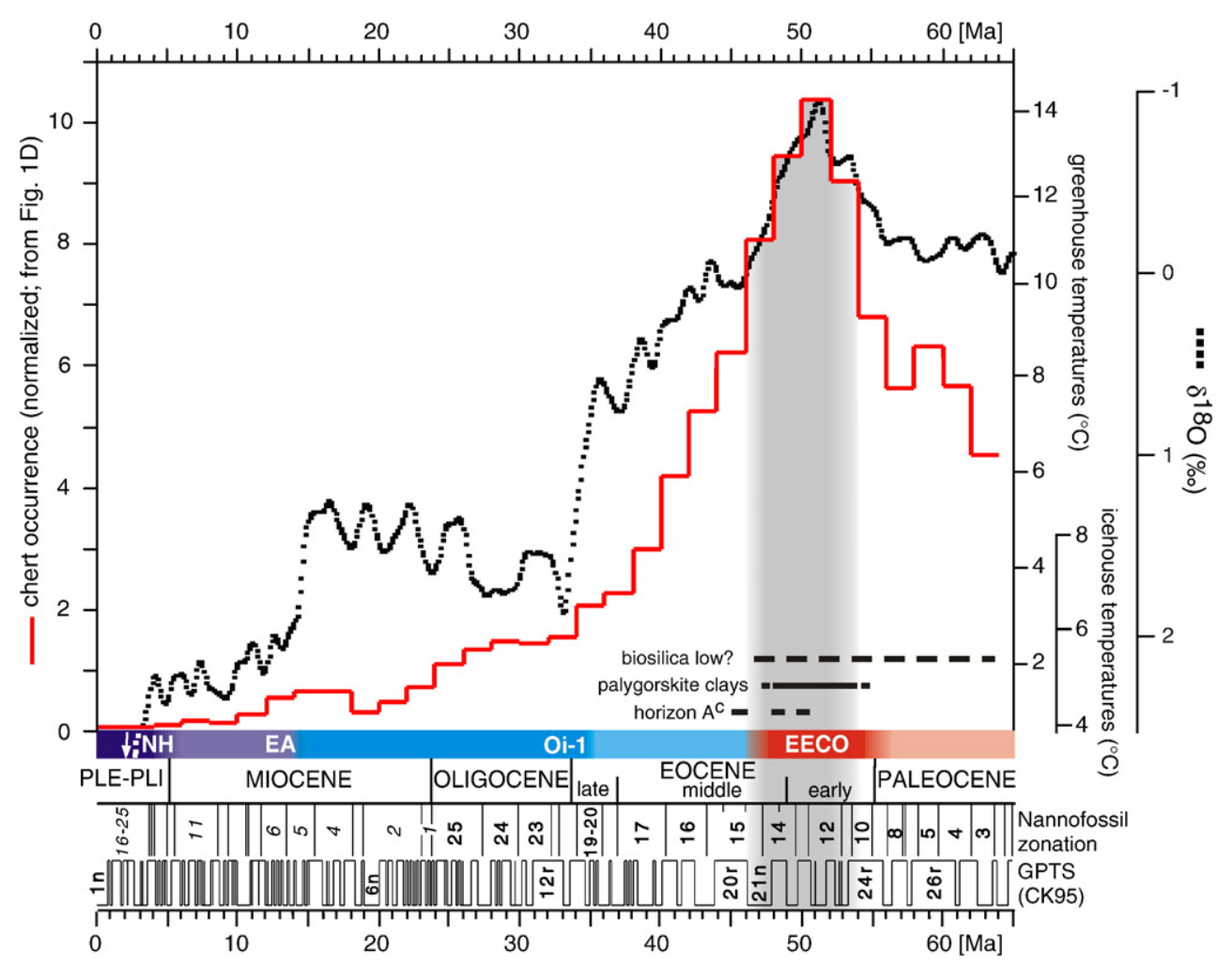

Fig. 2. Global chert occurrences corrected for the uneven chronostratigraphic distribution of DSDP and ODP holes and expressed as \% of the total number of chert intervals per hole present in the 0-64 Ma interval (from Fig. 1D) compared to the benthic oxygen isotope-based deep-sea temperatures computed for a greenhouse (small to negligible ice sheets) and icehouse (large ice sheets) worlds (Miller et al., 2005). The early Eocene peak of chert occurrence correlates to maximum deep-sea temperatures of the early Eocene climatic optimum (EECO). Horizontal bars represent the stratigraphic ranges of acoustic horizon $\mathrm{A}^{\mathrm{c}}$, the presumed minimum in radiolarian and diatom occurrences inferred from DSDP and ODP data (Fig. 1C) and palygorskite clays from the western Central Atlantic and the Gulf of Guinea (from Pletsch, 1998; 2001). Oi-1 is the earliest Oligocene glaciation, EA is the onset of the East Antarctic ice-sheet, and NH is the onset of Northern Hemisphere ice-sheets (Zachos et al., 2001). Ages and nannofossil zonation (NP Zones in bold and NN Zones in italics; Martini, 1971) are from Berggren et al. (1995) using geomagnetic polarity time scale (CK95) from Cande and Kent (1995).

warm conditions of EECO. As bottom water temperatures cooled over the ensuing $\sim 15 \mathrm{Myr}$ of the middle and late Eocene, chert occurrences also decreased to less than $1 / 4$ of peak values starting at the earliest Oligocene Oi-1 glaciation at $\sim 34 \mathrm{Ma}$. According to the compilation of Hein and Parrish (1987), no analogues of bedded cherts exist in the modern ocean sedimentary record. Higher (lower) chert occurrences thus correspond to warmer (cooler) bottom water temperatures, suggesting that primary paleoclimatic factors rather than secondary diagenetic processes control chert formation.

The early Eocene statistical peak in chert occurrences in DSDP and ODP cores is confirmed from several wellstudied outcrop sections correlated magneto-biostratigraphically to the Berggren et al. (1995) time scale (Fig. 3). The classic sections of the Gubbio Apennines, Italy, were deposited at a paleolatitude of $\sim 35^{\circ} \mathrm{N}$ in a pelagic environment of the Tethys Ocean. In the Bottaccione section, the base of the upper cherty member of the Red Scaglia is placed within magnetochron C24n and nannofossil Zone NP12 (Napoleone et al., 1983; Monechi and Thierstein, 1985). In the Contessa Highway section, the base of the same member is placed at the $\mathrm{C} 23 \mathrm{r}-\mathrm{C} 23 \mathrm{n}$ magnetochron boundary within Zone NP12 (Lowrie et al., 1982; Monechi and Thierstein, 1985), whereas in the nearby Contessa Road section, it is placed close to the $\mathrm{C} 24 \mathrm{n}-\mathrm{C} 23 \mathrm{r}$ magnetochron boundary (Lowrie et al., 1982). Further inspection of Fig. 3 reveals that the decline in chert occurrence in the Gubbio Apennines seems to be constrained to the younger part of magnetochron C21r, within Zone NP14 ( 48 Ma).

These data from pelagic sections are in substantial agreement with data from classic shallow-water land outcrop sections from the U.S. Atlantic and Gulf Coastal Plains and elsewhere, for example, the opaline claystones of the Black Mingo Formation of South Carolina of early-middle Eocene age (Weaver and Wise, 1974) and the chert-bearing Tallahatta Formation of Alabama 


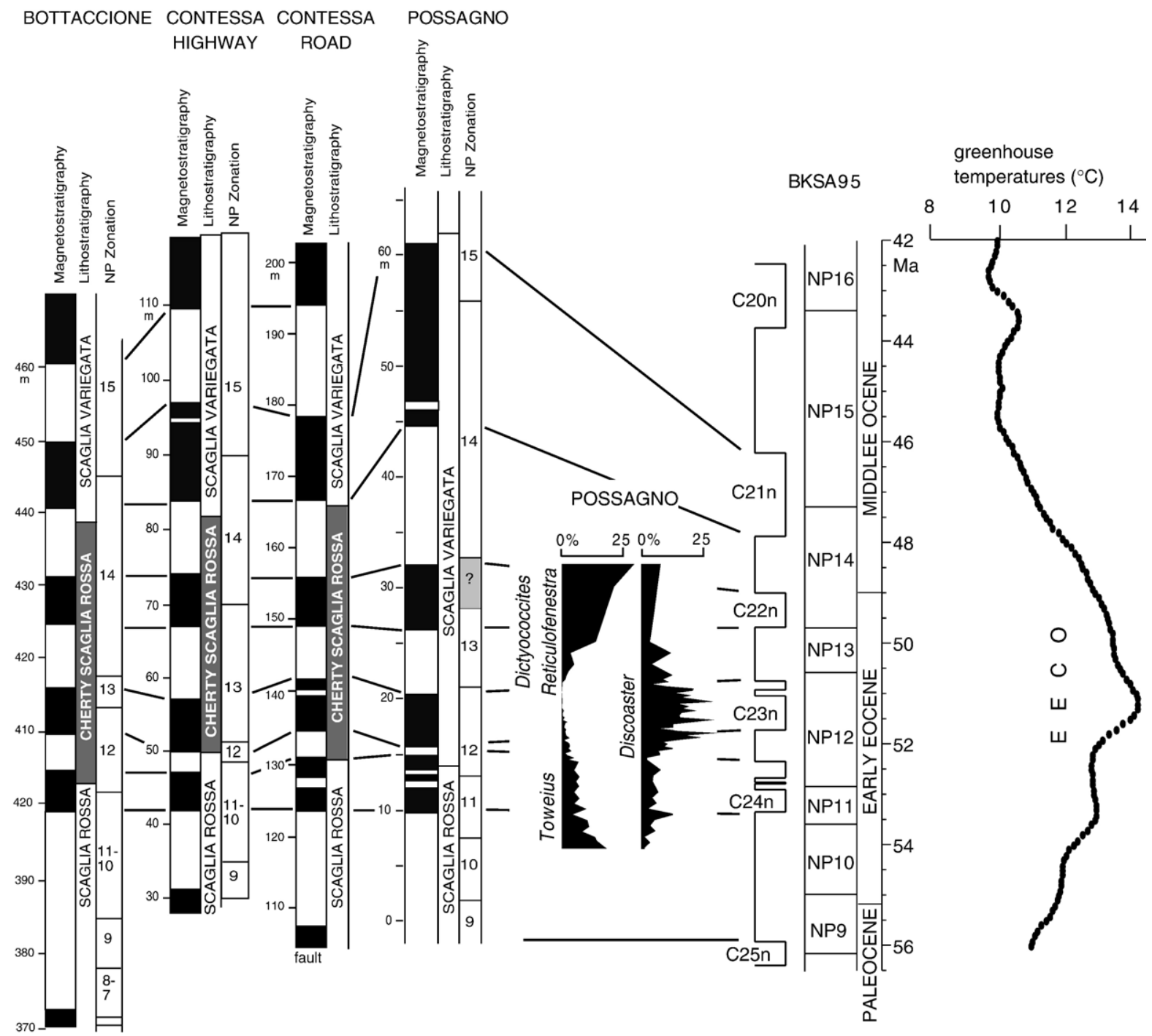

Fig. 3. Eocene Tethyan sections from the northern Apennines at Bottaccione, Contessa Highway, and Contessa Road (Lowrie et al., 1982; Napoleone et al., 1983; Monechi and Thierstein, 1985) and the Possagno section from the southern Italian Alps (Agnini et al., 2006) correlated to the Berggren et al. (1995) time scale and the benthic oxygen isotope-based bottom water temperatures computed for a greenhouse world (Miller et al., 2005). The base of the cherty member of the Scaglia Rossa in the northern Apennines is constrained to the C24n (top) $-\mathrm{C} 23 \mathrm{n}$ (base) magnetochron interval $(\sim 52 \mathrm{Ma})$ within nannofossil Zone NP12, whereas the top of the cherty member is constrained to magnetochron C21r (top) within Zone NP14 ( $\sim 48 \mathrm{Ma}$ ). Stratigraphic levels of $\sim \mathrm{C} 23$ age at Possagno are characterized by an oligotrophic event (represented mainly by a Discoaster Acme) broadly correlative to the occurrence of cherts in the Apennines sections and to maximum bottom water temperatures of the EECO.

(Wise and Weaver, 1973) of Zones NP12-NP14 age (Bybell and Gibson, 1983), or in Egypt where the chert-bearing Thebes Formation is of foraminifera Zones P8-P9 ( top of NP12-base of NP14) age (Berggren and Ouda, 2003). There is, thus, general agreement that the onset and termination of peak chert occurrences are consistent in very different paleoenvironmental settings and occur over a common time interval ( $\sim 54-46 \mathrm{Ma}$ from statistical analysis of DSDP and ODP data; $\sim 52-48 \mathrm{Ma}$ in key land outcrop sections) that closely coincides with EECO warm bottom water temperatures.

The reconstructed geographic locations of DSDP and ODP sites that document the peak early Eocene chert occurrences at $\sim 54-46 \mathrm{Ma}$ are broadly dispersed (Fig. 4). In the North Atlantic region, there is a wide swath of occurrences that range from tropical latitudes (e.g., DSDP Site 98 ; latitude $\sim 23^{\circ} \mathrm{N}$ ) to moderately high latitudes (e.g., DSDP Site 552; latitude $\sim 51^{\circ} \mathrm{N}$ ), and more generally over the broad area of the western North 


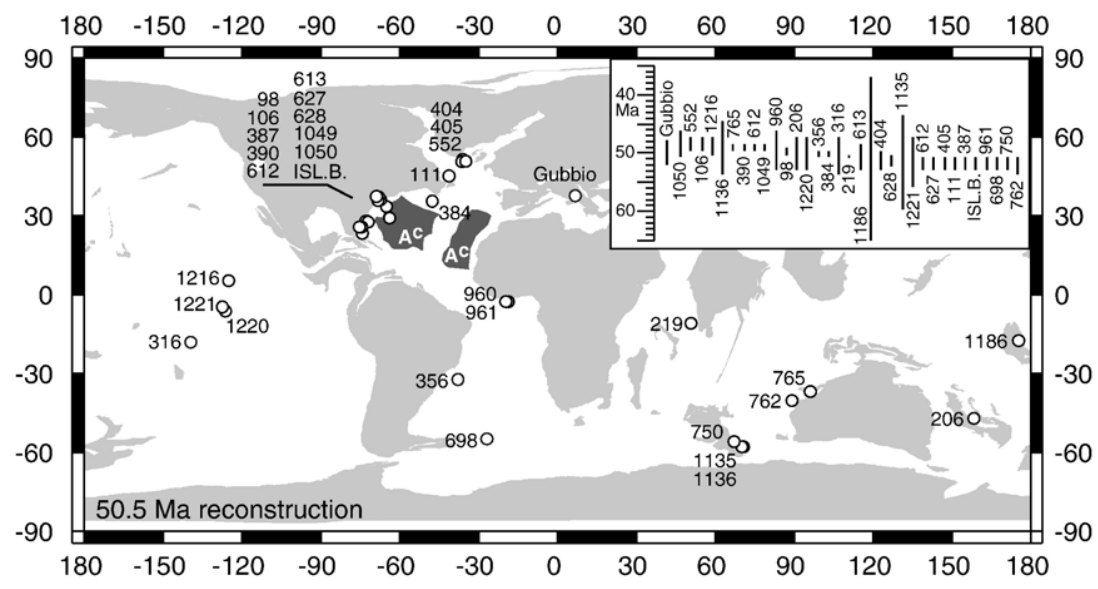

Fig. 4. Paleogeographic reconstruction at 50.5 Ma (Ocean Drilling Stratigraphic Network online; plate reconstruction data from Hay et al., 1999 and references therein) showing the dispersed locations of DSDP and ODP sites that define the peak early Eocene chert occurrence at $~ 54-46$ Ma (Zones NP11-NP15a); see the inset and Table 1 for the actual temporal distribution of chert intervals. Shaded grey regions are the inferred extents of acoustic horizon $\mathrm{A}^{\mathrm{c}}$.

Atlantic where acoustic horizon $\mathrm{A}^{\mathrm{c}}$ was traced and correlated to a chert layer of Zone NP13 age at DSDP Site 384 (J-anomaly ridge), to core catcher cherts of inferred Zone NP14 age at DSDP Site 385 (Voegel seamount), to the top of calcareous turbidites of Zones NP15a-NP15b boundary age at DSDP Site 386 (central Bermuda Rise), and to the top of a chert interval of Zone NP15b age at DSDP Site 387 (central Bermuda Rise) (Premoli Silva and Boersma, 1986; Aubry, 1995), although horizon $\mathrm{A}^{\mathrm{c}}$ seems to correspond to an erosional event in ODP Sites 1049 to 1052 from the Blake Nose (Norris et al., 2001). The widely dispersed middle to high latitude chert occurrences that characterize the North Atlantic region are not obviously compatible with the expected loci of upwelling regions, even as drawn by Ramsay (1971). The North Atlantic chert distribution may have been conceivably controlled by mid-latitude coastal upwelling, which is however usually restricted to the eastern side of subtropical gyres (e.g., Seibold and Berger, 1993; Huber et al., 2004), making the occurrence of cherts along the western margin of the North Atlantic (e.g., U.S. Atlantic coastal plain, shelf and slope; Bermuda Rise) rather unexpected as a proxy of upwelling.

Outside the North Atlantic, occurrences in subequatorial tropics and high southern latitudes may track the locations of equatorial and circum-polar upwelling zones (Fig. 4). A belt of high biogenic productivity associated with the paleoequator was present in the Pacific Ocean throughout most of the Cenozoic although it was weak and diffuse during the early Eocene (Moore et al., 2002; 2004; Pares and Moore, 2005); this seems to suggest that the Pacific Ocean, with large fetch effects, may have always had an important component of upwelling even during periods of reduced zonality.

\section{Comparison to 'silica burp' model}

McGowran (1989) proposed the "silica burp" model to explain the origin for the North Atlantic chert anomaly, as follows. During the global warming leading to EECO, large amounts of dissolved silica from deep weathering, possibly enhanced by silica from volcanism in the North Atlantic Tertiary igneous province, accumulated in a warm, supposedly sluggish ocean. During the subsequent middle Eocene global cooling, more vigorous oceanic circulation and consequent upwelling made this silica reservoir available for enhanced biosilicification and triggered the deposition of biosiliceous precursors of chert across a broad high productivity zone extending in the North Atlantic up to at least $\sim 30^{\circ} \mathrm{N}$ (Ramsay, 1971) and generally corresponding to the cherts of acoustic horizon $\mathrm{A}^{\mathrm{c}}$.

Recently acquired data seem to confirm this vision of an early Eocene ocean enriched in dissolved silica and characterized by less focused upwelling of deep water. Backtracking of DSDP and ODP drill sites on the Pacific plate shows that biosiliceous sedimentation associated with the equatorial divergence was low and smeared over a broad band of $\sim 15^{\circ}$ latitude in the early Eocene (Moore et al., 2002; 2004; Pares and Moore, 2005). In a world where polar regions were warm and mostly ice-free, the latitudinal temperature gradient was reduced relative to the present (by an amount still being debated and depending critically on estimates of tropical sea surface temperatures; see Schrag, 1999; Zachos et al., 2001; 
Pearson et al., 2001; Zachos et al., 2002; Pearson et al., 2002). As a consequence, zonal wind intensities were lower than at present, as inferred from the eolian record in deep-sea sediments (e.g., Rea, 1994), and presumably also the wind-driven ocean circulation and associated upwelling. In this respect, the isotopic evidence of warmer than present tropical oceans (Pearson et al., 2001) is consistent with indirect evidence of reduced upwelling of deep water during this time interval (Cervato and Burckle, 2003). Warmer temperatures, however, do not necessarily imply the development of a stagnant ocean (e.g., de Boer et al., 2007), and indeed, the apparent absence of extensive organic-rich sediments would seem to indicate that some degree of bottom water ventilation was operative in the early Eocene greenhouse world. In any case, bottom water temperatures were much higher in EECO than present (up to $14{ }^{\circ} \mathrm{C}$; Zachos et al., 2001; Miller et al., 2005), and thus so were rates of silica dissolution (Erez et al., 1982), implying that silica from the enhanced weathering of silicates due to an accelerated hydrological cycle, or from other sources, could easily build up in the oceans.

As McGowran (1989) suggested, the oceans became better stirred later in the Eocene when the supposed biosiliceous precursors of horizon $\mathrm{A}^{\mathrm{c}}$ chert were deposited and buried. Backtracked DSDP and ODP sites of the Pacific plate show high deposition rates of biosiliceous sediments more closely related to the equatorial belt at least since the Oligocene (Moore et al., 2002; 2004; Pares and Moore, 2005), a mode of silica removal similar to that operating in modern oceans and largely due to a vigorous wind-driven surface ocean circulation and the sinking of cold saline waters in polar regions.

Our new assessment of chert occurrences contradicts the 'silica burp' model in a critical and robust way: the Eocene chert peak straddles EECO rather than falling on the subsequent global cooling in the middle Eocene, suggesting that chert formation was tightly coupled to peak greenhouse conditions rather than to the onset of icehouse conditions (Fig. 2). As already pointed out by Yool and Tyrell (2005), a long-term decoupling of silica input during the early Eocene warming and output mainly during the middle Eocene (as biosiliceous precursors of horizon $\mathrm{A}^{\mathrm{c}}$ chert) is unlikely because geochemical modeling of the silica cycle suggests that on timescales greater than a few hundred thousand years, silica input and output should be closely balanced for any given equilibrium temperature. The fact that the ocean in the late Paleocene and early Eocene was enriched in dissolved silica thus cannot represent a precondition for a biosilicification event that occurred millions of years afterwards. Our analysis, on the other hand, shows that peak cherts occurred in a time interval that closely coincides with EECO. In this respect, it seems likely that acoustic horizon $\mathrm{A}^{\mathrm{c}}$ does not represent an extended postEECO chert deposit as envisaged by McGowran (1989) but rather marks either the upper level of the peak cherts or just some seismically reflective regional concentration of chert at discrete stratigraphic levels within the general distribution of EECO peak cherts (Fig. 2).

A further problem with the "silica burp" model is that the biosiliceous precursors of EECO peak cherts were not closely tied to expected loci of equatorial, polar, or even coastal upwelling in the North Atlantic (Fig. 4). Therefore, it is problematic how the cherts resulted from enhanced biosilicification due to more vigorous oceanic circulation as suggested by McGowran (1989). Oceanic divergences were in fact poorly expressed, even in the Pacific, in the warm and less wind-driven early Eocene ocean (Moore et al., 2002; 2004; Pares and Moore, 2005). Hence, it is not clear how predominantly biosiliceous productivity could have removed the abundant silica that entered the ocean in the warm climate of EECO.

\section{New EECO chert model}

We propose the following scenario to account for the formation of EECO cherts. During the extreme warmth of the early Eocene, abundant silica entered the ocean. Silica may have been derived from enhanced continental weathering and runoff in a warmer and more humid climate (Sloan and Rea, 1995; Zachos et al., 2001; Miller et al., 2005) that may have been due to the buildup of greenhouse gases (e.g., Owen and Rea, 1985; Pearson and Palmer, 2000; Royer et al., 2007; but see Sinha and Stott, 1994; Royer et al., 2001).

For the silica cycle to remain in equilibrium, the same amount of silica coming into the ocean had to be expelled and sequestered in sediments over the residence time scales of silica (Yool and Tyrell, 2005). Yet, at the same time, biosiliceous productivity in the open ocean was low and geographically dispersed because upwelling efficiency along divergences was reduced due to decreased winds (Rea, 1994). Inspired by puzzling reports of only very rare radiolarians associated with abundant Eocene cherts (Gartner, 1970; Wise and Weaver, 1974; Premoli Silva and Boersma, 1986; Sanfilippo and Nigrini, 1998), we interpret the decreased number of biosiliceous fossil occurrences in DSDP-ODP holes in the $\sim 46$ to $52 \mathrm{Ma}$ time interval, corresponding at the older end to an apparently global interval of deep sea sediments with very sparse and poorly preserved radiolarian fauna (Sanfilippo and Nigrini, 1998), as evidence for decreased biosiliceous productivity in the open ocean rather than increased 
dissolution and diagenesis of radiolarians and diatoms at about the time of EECO peak cherts (Figs. 1, 2). Cervato and Burckle (2003) noticed that diatom speciations and extinctions are apparently absent from tropical latitudes during the early Cenozoic (and Cretaceous), and also attributed this to reduced equatorial upwelling and low productivity.

The paradoxical observation of abundant chert associated with low biosiliceous productivity, albeit potentially contaminated by several sources of bias in the database (Spencer-Cervato, 1999), receives further confirmation by studies on the evolutionary trends of planktonic and benthic foraminifera through the Paleogene that indicate expanded oligotrophy (low productivity) throughout the open ocean and on adjacent margins in the early Eocene (e.g., Hallock et al., 1991). Furthermore, recent magneto-biostratigraphic data from a land section at Possagno in the southern Italian Alps (Agnini et al., 2006) indicate that stratigraphic levels of around chron $\mathrm{C} 23$ age $(\sim 52-50 \mathrm{Ma})$, correlative to the cherty unit of the Gubbio Apennines and to maximum EECO deep-sea temperatures, are characterized by a Discoaster Acme, a warm-water taxon that thrived in oligotrophic conditions, and the virtual absence of taxa typical of colder and more eutrophic waters (Fig. 3; Agnini et al., 2006 and references therein). Hollis et al. (2005) reached similar conclusions concerning low productivity (oligotrophic) conditions during the EECO for the Mead Stream land section of New Zealand. These data seem to indicate that biosiliceous productivity, usually considered the dominant mode of silica removal (e.g., Kidder and Erwin, 2001), may have been inadequate to compensate for the enhanced amounts of silica entering the oceans during EECO.

We suggest that silica precipitation for cherts forming during the EECO received an inorganic assist from clay minerals, which can take up dissolved silica from silicaenriched waters and form more siliceous phases (Mackenzie et al., 1967; Broecker, 1971). Clay minerals are common in association with deep-sea cherts (Lancelot, 1973). In particular, Pletsch $(1998,2001)$ noted the presence of authigenic palygorskite clays in chert-bearing sediments of early Eocene age from the western Central Atlantic and the Gulf of Guinea and speculated that warm, saline and silica-rich bottom waters favored their formation. Following Mackenzie et al. (1967), we suggest that the excess of silica that could not be absorbed by clay mineral precursors to form palygorskite precipitated directly as opal, with $\mathrm{Mg}$-solid compounds possibly acting as precipitation catalysts (Hinman, 1998).

Basin-basin fractionation by deep-sea circulation could help explain the high concentration of EECO chert occurrences in the North Atlantic. Berger (1970) suggested that deep waters enriched in silica and other nutrients and depleted in oxygen are transferred from a lagoonal-type basin to an estuarine-type basin. For example, the modern ocean conveyor circulation consists of a North Atlantic lagoonal basin (silica and nutrientdepleted and oxygen-enriched) that transfers deep waters to a Pacific estuarine basin (silica and nutrient-enriched and oxygen-depleted). Berger (1970) pointed out that if the estuarine basin is small with respect to the lagoonal basin that feeds it, silica could become highly concentrated in the estuarine basin. We speculate for the existence of a reverse conveyor belt circulation in the early Eocene, whereby a small North Atlantic estuarine basin was at the end of a far-traveled flow deep waters from other basins. Thomas (2004) provided neodymium isotopic evidence for deep-water production in the North Pacific during the Paleocene-early middle Eocene $(\sim 65-45 \mathrm{Ma})$ and it is also possible that the North Atlantic was estuarine with respect to the Tethys, from which it received an inflow of warm saline deep waters (e.g., Pak and Miller, 1992). Such a reverse circulation model might help to explain the great concentration of chert in the silica-enriched North Atlantic of the early Eocene. In addition, the general absence of extensive early Eocene anoxic sediments in the North Atlantic can be taken as indirect confirmation that (biosiliceous) productivity was low and hence potentially incapable of balancing completely the silica cycle; this is because deep waters flowing into an estuarine basin tend to bring nutrients, which sustain productivity, but little oxygen, which would inhibit the combustion of organic matter and thereby promote the formation of anoxic sediments (Berger, 1970). In contrast, inorganic modes of silica precipitation could have taken place on the sea floor and/or within the sediment wherever the appropriate local conditions were met (e.g., availability of detrital clay mineral precursors, sufficient silica concentrations, etc.), which would explain why North Atlantic EECO cherts were latitudinally and bathymetrically dispersed and not confined to obvious upwelling zones.

In post-early Eocene times, chert occurrences decreased as global climate shifted toward cooler states and the oceans became better stirred and more productive (Miller et al., 2005). According to Pearson et al. (2007), the long-term Eocene cooling trend occurred mainly in polar regions and had little affect in the tropics, which would imply a higher latitudinal temperature gradient and a more vigorous wind-driven ocean circulation. A major radiolarian turnover was observed in ODP Leg 171 (Blake Nose, western North Atlantic) sediments close to the early-middle Eocene boundary (Sanfilippo and Blome, 2001) and this could mark the strengthening of upwelling 
circulation and biosiliceous productivity. Indeed, Norris et al. (2001) suggested that unconformities close to the early-middle Eocene boundary, and which in the western North Atlantic were tentatively correlated to horizon $\mathrm{A}^{\mathrm{c}}$, may represent a global change to more vigorous deepwater circulation, which, according to our analysis (Fig. 2), would coincide with the decrease in chert occurrences rather than triggering chert formation. Since then, bottom waters have been evidently too cold for direct chert formation (or even for the transformation of biosiliceous sediments into chert) and the more vigorous ocean circulation promoted the generation of biogenic silica along focused divergences, determining the biogenic control on the silica cycle as we know it from modern day oceans.

\section{Conclusions}

According to the present analysis, two modes of silica burial may have operated alternatively in the geologic record, by way of inorganic chemical precipitation or by biogenic silica deposition. Either mode of silica formation responds to the obligation of the ocean system to expel in sediments an amount of silica equivalent to the silicic acid supplied even on relatively short time scales. Paraphrasing Yool and Tyrell (2005), the relative rapidity of the silica burial response suggests that the temporal distribution of silica input to the ocean may be inferred from the sedimentary record whereby high (low) rates of silica burial correspond to high (low) rates of silica input from land weathering and/or volcanism.

In the early Eocene, basin-basin fractionation by deepsea circulation could have raised the concentration of EECO dissolved silica especially in a relatively small North Atlantic estuarine basin, where the burial of mainly authigenic (primary) chert and Si-saturated clays (as well as some biogenic silica, e.g., Weaver and Wise, 1974) did not occur strictly along oceanic upwelling belts but corresponded to high rates of input of silica to an upwelling-deficient ocean from enhanced chemical land weathering and volcanism under severe greenhouse conditions, which show analogies with greenhouse intervals of the Late Cretaceous (e.g., Steuber et al., 2005). The post-Eocene cooling trend and onset of major glaciation with an associated long-term increase in physical weathering correlate with biogenic opaline deposition at the bottom of cold oceans under focused oceanic divergences, where diagenetic reactions taking place after burial are responsible for the time-promoted and generally slow conversion of biosilica into chert. This should be the case also of cherts from biosiliceous oozes originally deposited at near-equatorial paleolatitudes in a mid-late Jurassic ocean (Muttoni et al., 2005), which can be regarded as cooler relative to other JurassicCretaceous greenhouse periods (Frakes and Francis, 1988) and where an enhanced input of silica was likely derived from weathering and/or volcanism due to ocean opening and break-up of Pangea in the Jurassic. Inorganic chert formation therefore acts as a silica regulator mainly (only?) under extreme greenhouse conditions when ocean circulation becomes upwelling-deficient and incapable to sustain a siliceous biomass adequate to level off, upon burial, the input of silica to the ocean. This would explain why there are no modern examples of inorganic chert formation because in cold and well-stirred oceans the role of silica regulation is efficiently controlled by biosiliceous productivity and dominated from the Oligocene by diatoms (e.g., Cervato and Burckle, 2003), perhaps because of enhanced nutrient supply to the photic zone from below in upwelling zones and from above by eolian sources.

A final question remains, how can the two modes of chert formation described above be recognized before interpreting cherts in the geologic record as paleolatitude, paleoceanographic or climate proxies. A rough criterion could be the occurrence of abundant radiolarians or diatoms, past witnesses of focused upwelling circulation in well-mixed cold oceans. However, the Eocene versus Jurassic scenarios of chert formation illustrated here strongly suggests that reliance would be better placed on paleomagnetism as a means to determine paleolatitudes.

\section{Acknowledgments}

We thank Cinzia Cervato and Josh Reed for providing the number of DSDP and ODP holes and the number of Radiolaria, diatom and nannoplankton occurrences from the Neptune database, Claudia Agnini for revising the biostratigraphic attribution of some chert intervals in the literature, Lloyd Burckle, Luca Lanci, S. Galeotti, Ted Moore, Ken Miller, Bill Ryan and Isabella Premoli Silva for insightful comments on various stages of analysis and manuscript preparation, and David L. Kidder and an anonymous reviewer for constructive criticisms that allowed us to improve the submitted paper. LDEO contribution \#7040.

\section{References}

Agnini, C., Muttoni, G., Kent, D.V., Rio, D., 2006. Eocene biostratigraphy and magnetic stratigraphy from Possagno, Italy: the calcareous nannofossil response to climate variability. Earth and Planetary Science Letters 241, 815-830. 
Aubry, M.-P., 1995. From chronology to stratigraphy: interpreting the lower and middle Eocene stratigraphic record in the Atlantic Ocean. In: Berggren, W.A., Kent, D.V., Aubry, M.-P., Hardenbol, J. (Eds.), Geochronology, Time Scales and Global Stratigraphic Correlations. SEPM Special Publication, vol. 54, pp. 215-274.

Berger, W.H., 1970. Biogenous deep-sea sediments: fractionation by deep-sea circulation. Geological Society of America Bulletin 81, 1385-1402.

Berger, W.H., Winterer, E.L., 1974. Plate stratigraphy and the fluctuating carbonate line. In: Hsu, K.J., Jenkins, H.C. (Eds.), Pelagic Sediments on Land and under the Sea. International Association of Sedimentologists, pp. 11-48.

Berggren, W.A., Ouda, K., 2003. Upper Paleocene-lower Eocene planktonic biostratigraphy of the Dababiya section, Upper Nile Valley (Egypt). Micropaleontology 49, 61-92.

Berggren, W.A., Kent, D.V., Swisher, C.C., Aubry, M.P., 1995. A revised Cenozoic geochronology and chronostratigraphy. In: Berggren, W.A., Kent, D.V., Aubry, M.-P., Hardenbol, J. (Eds.), Geochronology, Time Scales and Global Stratigraphic Correlations. SEPM Special Publication, vol. 54, pp. 129-212.

Broecker, W.S., 1971. A kinetic model for the chemical composition of sea water. Quaternary Research 1, 188-207.

Bybell, L.M., Gibson, T.G., 1983. Biostratigraphy of Tallahatta Formation (Eocene) in Eastern Gulf Coastal Plain and revised age for Claiborne Stage. American Association of Petroleum Geologists Bulletin 67 (9), 1462.

Cande, S.C., Kent, D.V., 1995. Revised calibration of the geomagnetic polarity time scale for the Late Cretaceous and Cenozoic. Journal of Geophysical Research 100, 6093-6095.

Cervato, C., Burckle, L., 2003. Pattern of first and last appearance in diatoms: oceanic circulation and the position of polar fronts during the Cenozoic. Paleoceanography 18 (2), 1055.

de Boer, A.M., Sigman, D.M., Toggweiler, J.R., Russell, J.L., 2007. Effect of global ocean temperature change on deep ocean ventilation. Paleoceanography 22, PA2210. doi:10.1029/ 2005PA001242.

Erez, J., Takahashi, K., Honjo, S., 1982. In-situ dissolution experiment of radiolaria in the central North Pacific ocean. Earth and Planetary Science Letters 59 (2), 245-254.

Frakes, L.A., Francis, J.E., 1988. A guide to Phanerozoic cold polar climates from high-latitude ice-rafting in the Cretaceous. Nature $333,547-549$.

Gartner, S., 1970. Sea-floor spreading, carbonate dissolution level, and the nature of Horizon A. Science 169, 1077-1079.

Gibson, T.G., Towe, K.M., 1971. Eocene volcanism and the origin of Horizon A. Science 172, 152-154.

Hagstrum, J.T., Murchey, B.L., 1993. Deposition of Franciscan Complex cherts along the paleoequator and accretion to the American margin at tropical paleolatitudes. Geological Society of America Bulletin 105 (6), 766-778.

Hallock, P., Premoli Silva, I., Boersma, A., 1991. Similarities between planktonic and larger foraminiferal evolutionary trends through Paleogene paleoceanographic changes. Palaeogeography, Palaeoclimatology, Palaeoecology 83 (1-3), 49-64.

Hay, W.W., DeConto, R., Wold, C.N., Wilson, K.M., Voigt, S., Schulz, M., Wold-Rossby, A., Dullo, W.-C., Ronov, A.B., Balukhovsky, A.N., Soeding, E., 1999. Alternative global Cretaceous paleogeography. In: Barreran, E., Johnson, C. (Eds.), The Evolution of Cretaceous Ocean/Climate Systems. Geological Society of America Special Paper, vol. 332, pp. 1-47.

Hein, J.R., Parrish, J.T., 1987. Distribution of siliceous deposits in space and time. In: Hein, J.R. (Ed.), Siliceous Sedimentary Rock-
Hosted Ores and Petroleum. Van Nostrand Reinhold Co., New York, pp. 10-57.

Hinman, N.W., 1998. Sequences of silica phase transitions: effects of $\mathrm{Na}, \mathrm{Mg}, \mathrm{K}, \mathrm{Al}$, and Fe ions. Marine Geology 147, 13-24.

Hollis, C.J., Dickens, G.R., Field, B.D., Jones, C.M., Strong, P.C., 2005. The Paleocene-Eocene transition at Mead Stream, New Zealand: a southern Pacific record of early Cenozoic global change. Palaeogeography, Palaeoclimatology, Palaeoecology 215, 313-343.

Huber, M., Brinkhuis, H., Stickley, C.E., Doos, K., Sluijs, A., Warnaar, J., Schellenberg, S.A., Williams, G., 2004. Eocene circulation of the Southern Ocean: was Antarctica kept warm by subtropical waters? Paleoceanography 19, PA4026.

Kidder, D.L., Erwin, D.H., 2001. Secular distribution of biogenic silica through the Phanerozoic: comparison of silica-replaced fossils and bedded cherts at the series level. Journal of Geology 109, 509-522.

Lancelot, Y., 1973. Chert and silica diagenesis in sediments from the central Pacific. Initial Reports of the Deep Sea Drilling Project 17, 377-405.

Larson, R.L., Steiner, M.B., Erba, E., Lancelot, Y., 1992. Paleolatitudes and tectonic reconstructions of the oldest portion of the Pacific Plate: a comparative study. Proceedings of the Ocean Drilling Program. Scientific Results 129, 615-631.

Lowrie, W., Alvarez, W., Napoleone, G., Perch-Nielsen, K., Premoli Silva, I., Toumarkine, M., 1982. Paleogene magnetic stratigraphy in Umbrian pelagic carbonate rocks: the Contessa sections, Gubbio. Geological Society of America Bulletin 93, 414-432.

Mackenzie, F.T., Garrels, R.M., Bricker, O.P., Bickley, F., 1967. Silica in sea water: control by silica minerals. Science 155, 1404-1405.

Martini, E., 1971. Standard Tertiary and Quaternary calcareous nannoplankton zonation. In: Farinacci, A. (Ed.), Proceedings of the Second Planktonic Conference. Tecnoscienza, Rome, pp. 739-785.

Mattson, P.H., Pessagno Jr., E.A., 1971. Caribbean Eocene volcanism and the extent of Horizon A. Science 174, 138-139.

McGowran, B., 1989. Silica burp in the Eocene ocean. Geology 17, $857-860$.

Miller, K.G., Wright, J.D., Browning, J.V., 2005. Visions of ice sheets in a greenhouse world. Marine Geology 217, 215-231.

Monechi, S., Thierstein, H.R., 1985. Late Cretaceous-Eocene nannofossil and magnetostratigraphic correlations near Gubbio, Italy. Marine Micropaleontology 9, 419-440.

Moore, T.C., Rea, D.K., Lyle, M., Liberty, L.M., 2002. Equatorial ocean circulation in an extremely warm climate. Paleoceanography 17 (1), 1005. doi:10.1029/2000PA000566.

Moore, T.C., Backman, J., Raffi, I., Nigrini, C., Sanfilippo, A., Pälike, H., Lyle, M., 2004. Paleogene tropical Pacific: clues to circulation, productivity, and plate motion. Paleoceanography 19, PA3013. doi:10.1029/2003PA000998.

Muttoni, G., Erba, E., Kent, D.V., Bachtadse, V., 2005. Mesozoic Alpine facies deposition as a result of past latitudinal plate motion. Nature 434, 59-63.

Napoleone, G., Premoli Silva, I., Heller, F., Cheli, P., Corezzi, S., Fischer, A.G., 1983. Eocene magnetic stratigraphy at Gubbio, Italy, and its implications for Paleogene geochronology. Geological Society of America Bulletin 94, 181-191.

Nigrini, C., Sanfilippo, A., Moore, T.J., 2006. Cenozoic radiolarian biostratigraphy: a magnetobiostratigraphic chronology of Cenozoic sequences from ODP Sites 1218, 1219, and 1220, equatorial Pacific. Proceedings of the Ocean Drilling Program. Scientific Results 199, 1-76.

Norris, R.D., Klaus, A., Kroon, D., 2001. Mid-Eocene deep water, the Late Paleocene Thermal Maximum and continental slope mass wasting during the Cretaceous-Paleogene impact. In: Kroon, D., 
Norris, R.D., Klaus, A. (Eds.), Western North Atlantic Paleogene and Cretaceous Paleoceanography. Geological Society of London, London, pp. 23-48.

Owen, R.M., Rea, D.K., 1985. Sea-floor hydrothermal activity links climate to tectonics: the Eocene carbon dioxide greenhouse. Science 227, 166-169.

Pak, D.K., Miller, K.G., 1992. Paleocene to Eocene benthic foraminiferal isotopes and assemblages: implications for deepwater circulation. Paleoceanography 7 (4), 405-422.

Pares, J.M., Moore, T.C., 2005. New evidence for the Hawaiian hotspot plume motion since the Eocene. Earth and Planetary Science Letters 237 (3-4), 951-959.

Pearson, P.N., Palmer, M.R., 2000. Atmospheric carbon dioxide concentrations over the past 60 million years. Nature 406, 695-699.

Pearson, P.N., Ditchfield, P.W., Singano, J., Harcourt-Brown, K.G., Nicholas, C.J., Olsson, R.K., Shackleton, N.J., Hall, M.A., 2001. Warm tropical sea surface temperatures in the Late Cretaceous and Eocene epochs. Nature 413, 481-488.

Pearson, P.N., Ditchfield, P., Shackleton, N.J., 2002. Palaeoclimatology (Communication arising): tropical temperatures in greenhouse episodes. Nature 419, 898.

Pearson, P.N., van Dongen, B.E., Nicholas, C.J., Pancost, R.D., Schouten, S., Singano, J.M., Wade, B.S., 2007. Stable warm tropical climate through the Eocene epoch. Geology 35, 211-214.

Pletsch, T., 1998. Origin of lower Eocene palygorskite clays on the Cote D'Ivoire-Ghana transform margin, eastern equatorial Atlantic. Proceedings of the Ocean Drilling Program. Scientific Results $159,141-156$.

Pletsch, T., 2001. Palaeoenvironmental implications of palygorskite clays in Eocene deep-water sediments from the western central Atlantic. In: Kroon, D., Norris, R.D., Klaus, A. (Eds.), Western North Atlantic Paleogene and Cretaceous Paleoceanography. Geological Society, London, Special Publication, vol. 183, pp. 307-316.

Premoli Silva, I., Boersma, A., 1986. In: Tucholke, B.E., Vogt, P.R. (Eds.), Paleogene Facies of the Western North Atlantic Ocean. The Geology of North America, Boulder CO, pp. 527-546.

Ramsay, A.T.S., 1971. Occurrence of biogenic siliceous sediments in the Atlantic Ocean. Nature 233, 115-117.

Rea, D.K., 1994. The paleoclimatic record provided by eolian deposition in the deep sea: The geologic history of wind. Reviews of Geophysics 32 (2), 159-195.

Richards, D., Butler, R.F., Harms, T.A., 1993. Paleomagnetism of the late Paleozoic Slide Mountain terrane, northern and central British Columbia. Canadian Journal of Earth Sciences 30, 1898-1913.

Riech, V., von Rad, U., 1979. Eocene porcellanites and Early Cretaceous cherts from the western North Atlantic. Initial Reports of the Deep Sea Drilling Project 43, 437-455.

Royer, D.L., Wing, S.L., Beerling, D.J., Jolley, D.W., Koch, P.L., Hickey, L.J., Berner, R.A., 2001. Paleobotanical evidence for near present-day levels of atmospheric $\mathrm{CO}_{2}$ during part of the Tertiary. Science 292, 2310-2313.

Royer, D.L., Berner, R.A., Park, J., 2007. Climate sensitivity constrained by $\mathrm{CO}_{2}$ concentrations over the past 420 million years. Nature 446, 530-532.

Sanfilippo, A., Blome, C.D., 2001. Biostratigraphic implications of mid-latitude Palaeocene-Eocene radiolarian faunas from Hole 1051A, ODP Leg 171B, Blake Nose, western North Atlantic. In: Kroon, D., Norris, R.D., Klaus, A. (Eds.), Western North Atlantic Palaeogene and Cretaceous Palaeoceanography. Geological Society, London, Special Publication, vol. 183, pp. 185-224.
Sanfilippo, A., Nigrini, C., 1998. Upper Paleocene-Lower Eocene deep-sea radiolarian stratigraphy and the Paleocene/Eocene series boundary. In: Aubry, M.-P., Lucas, S.G., Berggren, W.A. (Eds.), Late Paleocene-Early Eocene Climatic and Biotic Events in the Marine and Terrestrial Records. Columbia University Press, New York, pp. 244-276.

Schrag, D.P., 1999. Effects of diagenesis on the isotopic record of late Paleogene tropical sea surface temperatures. Chemical Geology $161(1-3), 215-224$.

Seibold, E., Berger, W.H., 1993. The Sea Floor - An Introduction to Marine Geology. Springer-Verlag. 356 pp.

Sinha, A., Stott, L.D., 1994. New atmospheric $p \mathrm{CO}_{2}$ estimates from palesols during the late Paleocene/early Eocene global warming interval. Global and Planetary Change 9, 297-307.

Sloan, L.C., Rea, D.K., 1995. Atmospheric carbon dioxide and early Eocene climate: a general circulation modeling sensitivity study. Palaeogeography, Palaeoclimatology, Palaeoecology 119 (3-4), 275-292.

Spencer-Cervato, C., 1998. Changing depth distribution of hiatuses during the Cenozoic. Paleoceanography 13, 178-182.

Spencer-Cervato, C., 1999. The Cenozoic deep sea microfossil record: explorations of the DSDP/ODP sample set using the Neptune database. Palaeontologia Electronica 2 (2) art. 4: 268 pp.

Steuber, T., Rauch, M., Masse, J.-P., Graaf, J., Malkoc, M., 2005. Lowlatitude seasonality of Cretaceous temperatures in warm and cold episodes. Nature 437, 1341-1344.

Thomas, D.J., 2004. Evidence for deep-water production in the North Pacific Ocean during the early Cenozoic warm interval. Nature 430, 65-68.

Tucholke, B.E., 1979. Relationship between acoustic stratigraphy and lithostratigraphy in the western North Atlantic basin. Initial Reports of the Deep Sea Drilling Project 43, 827-846.

Tucholke, B.E., Mountain, G.S., 1979. Seismic stratigraphy, lithostratigraphy and paleosedimentation patterns in the North Atlantic basin. In: Talwani, M., Hay, W., Ryan, W.B.F. (Eds.), Deep Drilling Results in the Atlantic Ocean: Continental Margins and Paleoenvironment. Maurice Ewing Symposium, vol. 3. American Geophysical Union, Washington, D.C., pp. 58-86.

Weaver, F.M., Wise Jr., S.W., 1974. Opaline sediments of the southeastern coastal plain and Horizon A: biogenic origin. Science 184 (4139), 899-901.

Wise Jr., S.W., Weaver, F.M., 1973. Origin of cristobalite-rich Tertiary sediments in the Atlantic and Gulf Coastal plain. Transactions Gulf Coast Association Geological Societies, vol. 23, pp. 305-323.

Wise Jr., S.W., Weaver, F.M., 1974. Chertification of oceanic sediments. In: Hsu, K.J., Jenkins, H.C. (Eds.), Pelagic Sediments on Land and under the Sea. International Association of Sedimentologists, pp. 301-326.

Yool, A., Tyrell, T., 2005. Implications for the history of Cenozoic opal deposition from a quantitative model. Palaeogeography, Palaeoclimatology, Palaeoecology 218, 239-255.

Zachos, J., Pagani, M.N., Sloan, L., Thomas, E., Billups, K., 2001. Trends, rhythms, and aberrations in global climate $65 \mathrm{Ma}$ to Present. Science 292, 686-693.

Zachos, J.C., Arthur, M.A., Bralower, T.J., Spero, H.J., 2002. Palaeoclimatology (Communication arising): tropical temperatures in greenhouse episodes. Nature 419, 897-898. 